\title{
A Phase Linearisation-based Modulation Signal Bispectrum for Analysing Cyclostationary Bearing Signals
}

\author{
Yuandong $\mathrm{Xu}^{\mathrm{a}}$, Chao $\mathrm{Fu}^{\mathrm{a}}$, Ning $\mathrm{Hu}^{\mathrm{b}}$, Baoshan Huang ${ }^{\mathrm{c}}$, Fengshou Gu ${ }^{\mathrm{a}}$, Andrew D. Ball ${ }^{\mathrm{a}}$ \\ ${ }^{a}$ Centre for Efficiency and Performance Engineering, University of Huddersfield, Huddersfield, UK HD1 3DH \\ yuandong.xu@hud.ac.uk (Y.Xu), f.gu@hud.ac.uk (F. Gu) \\ ${ }^{\mathrm{b}}$ School of Mechanical Engineering, Hebei University of Technology, Tianjin, China 300131 \\ c School of Industrial Automation, Beijing Institute of Technology Zhuhai, Zhuhai, China 266109
}

\begin{abstract}
Bearings are used as the most important load-carrying transmission components in the various machines, thus subjecting to a number of faults including wear, fatigue pitting, cracks and son. Fault detection and diagnosis of bearings can effectively prevent the machine from such typical failures and subsequent consequences. The faults in bearings can lead to the vibration signals exhibit cyclostationary characteristics due to the inevitably random phase noise (or slippage between bearing components). In this paper, a Phase Linearisation based Modulation Signal Bispectrum (PL-MSB) is proposed to tune up the cyclostationary bearing signal into a periodic waveform by linearizing the instantaneous phase of the narrow frequency band signals. In this way, the signal becomes more deterministic and MSB can be effectively applied to suppression noise and obtain accurate and robust diagnosis results. As a result, this fault detector can achieve high performance in characterising the nonstationary bearing vibration signals and hence diagnose the bearing faults even in the case of extremely low signal to noise ratio $(\mathrm{SNR}<-20 \mathrm{~dB})$, which is benchmarked by the method of conventional MSB in both simulation and experiment studies.
\end{abstract}

Keywords: Rolling Bearings, Cyclostationary Signals, Phase Linearisation, Modulation Signal Bispectrum, Fault Diagnosis

\section{Introduction}

Rolling bearings are used in almost all rotating machines to constrain movement, react load and minimise friction between contacting elements. Although such bearings are designed and manufactured to be highly reliable and have long lifetimes, they remain a common cause of machine breakdown, particularly after extensive operation. To prevent machine breakdowns, sophisticated fault detection and diagnosis of rolling bearings are needed.

A range of factors can contribute to premature bearing failure, including lubrication failure, excessive temperature and improper installation ${ }^{1,2}$. Approaches to the fault detection and diagnosis of bearings exist based on a variety of measured parameters, including lubricant wear debris ${ }^{3}$, temperature ${ }^{4}$, acoustic emission ${ }^{5,6}$, airborne acoustics ${ }^{7}$ and vibration ${ }^{8}$. Of these different methods vibration-based approaches remain the most extensively employed in condition monitoring of bearings, and they have been proven to be both efficient and effective in the detection, diagnosis and location of defects in rolling bearings ${ }^{1}$.

The impacting that occurs when a rolling element in a bearing comes into contact with a local defect (such as a crack or fatigue pit) can excite the resonance of the bearing, and the vibrations become modulation signals. The modulation phenomenon leads to the most profitable characteristic in the fault detection and diagnosis, which utilises the higher SNR parts of the raw signals. Demodulation analysis of bearing vibration signals has been investigated by many researchers as an effective condition indicating approach, with perhaps the most famous demodulation method being the envelope analysis 
technique developed by Darlow et al. ${ }^{9}$. In envelope analysis, the determination of the optimal frequency band(s) is the key challenge and researchers have explored the use of the Kurtogram ${ }^{10}$ based on the Short-time Fourier Transform (STFT) or Wavelet Transform (WT) to select automatically the optimum frequency band for analysis. The Kurtogram has been shown to be a powerful means of detecting and diagnosing bearing faults, but it can suffer from its sensitivity to the high levels of background noise and the presence of aperiodic impulses ${ }^{11,12}$. For these reasons, the Kurtogram approach can sometimes struggle to detect bearing faults at an early stage of their development. Spectral Correlation has been developed to investigate cyclostationary characteristics of signals by extracting periodic energy features, and it has been applied successfully to vibration signals for detecting and diagnosing bearing problems ${ }^{13-16}$. As such, spectral correlation is something of a milestone in nonstationary signal analysis, in particular for bearing vibration signals. Spectral Correlation is very effective at extracting energy oscillation information from cyclostationary signals. Aside from considering the characteristics of cyclostationary signals, another feasible method is to tune the cyclostationary signal into a periodic one via a process of nonlinear resampling into the angle domain. Many methods have been developed to analyse time varying signals, likely the most well known of which is Time Synchronous Averaging (TSA). TSA is extensively used to increase the Signal to Noise Ratio (SNR) of a signal, and it has been shown to achieve good fault detection and diagnosis in rotating machines ${ }^{17-19}$. TSA can be used to resample a vibration signal by using a tachometer reference and consequently the signal segments from different revolutions can be aligned and averaged in the shaft angle domain. In this way, phase errors of signals in the time domain can be significantly reduced in the angular domain, however in some circumstances tachometer signals are not available, and hence 'tacho-less' TSA techniques have been developed to overcome this deficiency ${ }^{20-23}$. The core problem of tacho-less approaches is to estimate accurately the instantaneous frequency / phase of the desired signals ${ }^{18,19,24}$, and hence to allow the target signal characteristics to be aligned in the angular domain. Then the large quantities of averaging can be done to suppress random noise.

Fault signatures of incipient faults are usually very weak and despite the noise suppression methods, another effective method is to enhance desired signals. Stochastic resonance (SR) can enhance the desired weak signals using the proper noise, which is nonlinear phenomenon of a Brownian particle bouncing between a bistable potential ${ }^{25}$. The conventional SR technique is difficult to be applied in non-stationary signals and $\mathrm{He}$ et al. ${ }^{26}$ proposed a new method called multi-scale SR spectrogram (MSSRS) to significantly improve the performance of SR with non-stationary transient signals in bearing defect diagnosis. A thorough review of SR in machine condition monitoring can be found in the reference ${ }^{25}$. In an attempt to extract incipient bearing defect information more reliably, Hou ${ }^{27}$ introduces harmonic wavelet transform (HWT) based demodulation technique in the fault diagnosis of rolling bearings. Cui et al. ${ }^{28}$ employs the wavelet transform and sparse component analysis to detect bearing faults, which outperforms the independent component analysis. The methods cannot achieve the accurate and effective diagnosis in the extremely poor signal quality. To improve the performance of the envelope analysis, $\mathrm{Xu}$ and co-authors developed an autocorrelated envelope method to diagnose bearings faults under a poor SNR ${ }^{29}$. Gu et al. ${ }^{30}$ introduced the Modulation Signal Bispectrum (MSB) to supress noise and demodulate the vibration signals, leading to a very capable means of the condition monitoring of a reciprocating compressor. Tian ${ }^{31}$ and Rehab ${ }^{32}$ extended Gu's approach and verified the use of the MSB to detect bearing faults in circumstances with very low signal to noise ratios (SNRs). The MSB method is designed to process periodic signals, and it cannot effectively demodulate cyclostationary elements such as the vibrations induced by the slippage that occurs between bearing elements in practical working conditions.

The random phase errors caused by the slippage between rolling bearing components can distort the linear phase increment into a nonlinear one. Inspired by the research into the processing of time varying signals, the random phase errors in bearing signals can be modified to decrease the influence of nonlinear phase variation. On the basis that the MSB is an outstanding demodulation method with a high noise suppression capability ${ }^{30,31,33-35}$, this paper presents the development and evaluation of a 
Phase Linearisation-based MSB (PL-MSB), specifically aimed at eliminating the adverse effects of random phase errors and background noise, and hence enabling more effective and efficient fault detection and diagnosis of rolling element bearings.

The paper is arranged as follows: Section 2 introduces the algorithm of the proposed PL-MSB, and the subsequent two sections present simulated and experimental evaluations of the proposed method when applied to the detection and diagnosis of incipient rolling bearing faults. Conclusions are drawn in Section 5.

\section{Methodology of the Phase Linearisation based Modulation Signal Bispectrum (PL-MSB)}

\subsection{Modulation Signal Bispectrum (MSB)}

The MSB has been investigated by the authors in ${ }^{31,33-35}$ to extract the modulating components in the vibration, acoustics, and instantaneous current signals for detecting and diagnosing faults in a wide range of mechanical and electrical machines. It has been shown that the MSB is highly effective in extracting incipient fault signatures from monitored data. The MSB originated from the conventional bispectrum by additionally considering the lower sideband to describe modulation signals more accurately. The definition of MSB is expressed as follows ${ }^{34}$

$$
B_{M S}\left(f_{x}, f_{c}\right)=E\left\langle X\left(f_{c}+f_{x}\right) X\left(f_{c}-f_{x}\right) X^{*}\left(f_{c}\right) X^{*}\left(f_{c}\right)\right\rangle
$$

where, $f_{c}$ and $f_{x}$ denote the coupled frequency components. $E\langle\quad\rangle$ is the ensemble average of MSB matrixes obtained from the multiple segments of the modulation signals. $X(f)=\int_{-\infty}^{+\infty} x(t) e^{-i 2 \pi f t} d t$ is the Fourier transform of the signal $x(t)$.

For a perfect periodically modulated signal, the phase of the MSB can be calculated from:

$$
\phi\left(f_{x}, f_{c}\right)=\phi\left(f_{c}+f_{x}\right)+\phi\left(f_{c}-f_{x}\right)-\phi\left(f_{c}\right)-\phi\left(f_{c}\right)
$$

When the components $f_{c}$ and $f_{x}$ are coupled by amplitude modulation (AM) or phase modulation (PM), the subsequent phase has the relationship as

$$
\begin{aligned}
& \phi\left(f_{c}+f_{x}\right)=\phi\left(f_{c}\right)+\phi\left(f_{x}\right) \\
& \phi\left(f_{c}-f_{x}\right)=\phi\left(f_{c}\right)-\phi\left(f_{x}\right)
\end{aligned}
$$

Consequently, the phase of the MSB phrase $X\left(f_{c}+f_{x}\right) X\left(f_{c}-f_{x}\right) X^{*}\left(f_{c}\right) X^{*}\left(f_{c}\right)$ will be zero for AM signals or $\pm \pi$ for PM signals. If the signal contains both AM and PM components, the final phase would be in the range of $(0, \pm \pi)$. This means that the MSB matrixes of different signal segments can be averaged to retain modulation characteristics. This averaging will suppress background noise and noncoupled components because their phases randomly vary between segments.

Similar to the normalisation way of the bicoherence, the coherence of the MSB can be defined as

$$
b_{M S}\left(f_{x}, f_{c}\right)=\frac{\left|B_{M S}\left(f_{x}, f_{c}\right)\right|^{2}}{E\left\langle\left|X\left(f_{c}\right) X^{*}\left(f_{c}\right)\right|^{2}\right\rangle E\left\langle\left|X\left(f_{c}+f_{x}\right) X\left(f_{c}-f_{x}\right)\right|^{2}\right\rangle}
$$

The MSB coherence allows to indicate the degree of coupling and noise suppression among the modulation components. The MSB coherence is in the range from 0 to 1 , which is independent of the amplitude of the coupled components in the frequency domain. If the coherence is close to 0 , it means there is no interaction between the components $f_{x}$ and $f_{c}$, either independent components or random noise. In contrast, the coherence value approaching to 1 denotes that the interaction between the components $f_{x}$ and $f_{c}$ is strong and slightly influenced by the noise. 
Thanks to the great efforts made by Randall and Antoni, the Spectral Correlation has been recognised a milestone in the cyclostationary signal analysis, especially bearing fault detection and diagnoses. The Spectral Correlation is defined as

$$
S_{x}(\alpha, f)=E\left\{X\left(f-\frac{\alpha}{2}\right) X^{*}\left(f+\frac{\alpha}{2}\right)\right\}
$$

where, $X(f)$ is the Fourier transform of the signal $x(n) ; X^{*}(f)$ is the conjugate of the $X(f) ; \alpha$ is the cyclic frequency. The Spectral Correlation is a cyclostationary signal analysis method. It calculates two directional Fourier transform of the generalised autocorrelation, which is a means to characterise the second order cyclostationarity. In another word, the generalised autocorrelation describes the energy oscillation of cyclostationary signals. Two steps including autocorrelation and 2D FFT are equivalent to the definition in Equation (5).

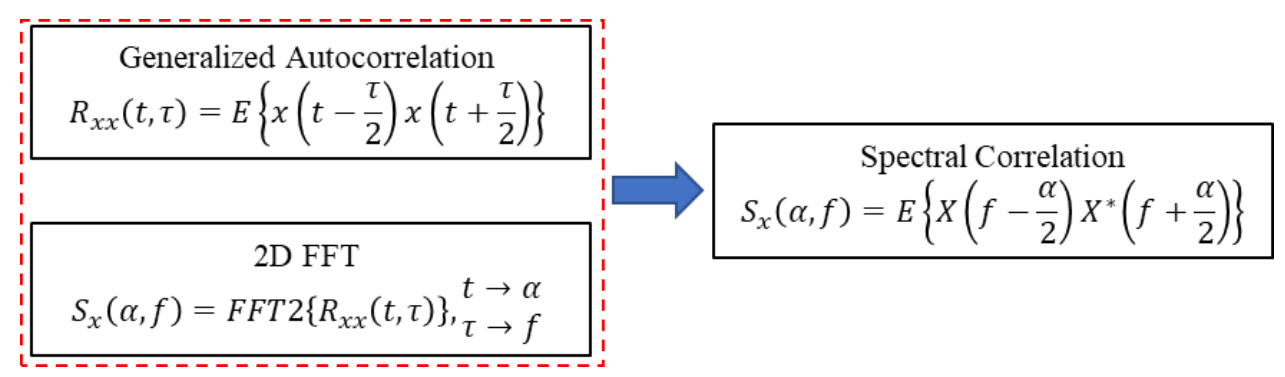

Figure 1 Theoretical background of Spectral Correlation

In contrast, the MSB analysis is still within the range of stationary signal analysis. It is based on the conventional Fourier transform, which aims to characterise the deterministic components in the modulation signals. The definitions of Spectral Correlation and MSB in Equation (1) and (5) are similar at the first glance but the theoretical background of these two methods are significantly different.

The high performance of the MSB has been demonstrated extensively in fault detection and diagnosis of bearings ${ }^{31}$, gearboxes ${ }^{33}$, motors ${ }^{34}$ and centrifugal pumps ${ }^{36}$. However, the implementation of the MSB is based on the conventional Fourier transform, which is not an efficient way to tackle cyclostationary signals. The random phase noise induced by the speed fluctuation and random slippage leads to the nonstationary signals, which goes beyond the capability of the Fourier transform. Consequently, the conventional Fourier transform based MSB is not as capable as the theoretical prediction in this circumstance. For this reason, this paper extends the MSB by using phase linearisation to tune nonlinear and nonstationary modulation signals. The improvement gained allows the PL-MSB to process the cyclostationary signals both efficiently and effectively.

\subsection{Phase Linearisation-based MSB (PL-MSB)}

As aforementioned, the developed approach in this paper aims to process the cyclostationary signals induced by the slippage between bearing components. The slippage in the rolling element bearings can be up to $2 \%{ }^{37}$ and the slippage makes the theoretically periodic impacts into a quasi-periodic series, terminologically named as cyclostationary signals.

The bearing fault signals are theoretically periodic for a purely rotating bearing with a localised fault under constant working conditions. Assume that the periodic fault vibration signal is comprised of multiple harmonic components, it can be expressed as

$$
s(t)=\sum_{k=0}^{\infty} A(k) \cos \left(k \omega_{0} t+\varphi_{k}\right)
$$

The fundamental frequency of the periodic signal is $\omega$ and the high order harmonics would be the integer times of the fundamental frequency but with different initial phase information. The harmonics around the natural frequencies of the bearing system would have a substantial amplitude compared with 
other harmonics. The $k$ th harmonic matching with the natural frequency is usually considered as the role of the carrier and the lower harmonics of $k-1, k-2, \ldots$ and upper harmonics of $k+1, k+2, \ldots$ are the sidebands around the carrier. These harmonics in a certain frequency bandwidth lead to a multiple component signal. The carrier can be seen as a monocomponent signal, which is described in the time and frequency domain by one single "ridge", representing the energy concentrated region. Such a monocomponent signal $s_{k}(t)$ can be represented by a complex form

$$
s_{k}(t)=A_{k}(t) e^{i \Phi_{k}(t)}
$$

where, $A_{k}(t)$ is the instantaneous amplitude. $\Phi_{k}(t)$ is the instantaneous phase, which is linearly incremental for this periodic signal. However, the phase of the signal is distorted by the random slippage. Consequently, the signal with the phase noise yields to

$$
s_{k}(t)=A_{k}(t) e^{i[\phi(t)+k n(t)]}
$$

where, $n(t)$ is the phase error. Then, the instantaneous phase of the nonstationary signal is

$$
\Phi_{k}(t)=\phi_{k}(t)+k n(t)
$$

The instantaneous phase can be divided into two parts. The deterministic phase $\phi(t)$ is a linear increment function of the time, which can be expressed as

$$
\phi_{k}(t)=k \omega_{0} t+\varphi_{k}
$$

where, $\varphi(0)$ is the initial phase of the periodic signal. Therefore, the instantaneous phase of the distorted periodic signal is

$$
\Phi_{k}(t)=k \omega_{0} t+\varphi_{k}+k n(t)
$$

The random error $n(t)$ is independent of the linear increment $\omega t$. A linear regression model can be used to fully explain the variation of the instantaneous phase and the phase noise $n(t)$ becomes the random deviations for the linear representation. A linear regression model can then be used to remove the standard errors. The estimate of the linear phase can be achieved by the least square method

$$
\widehat{\phi}_{k}(t)=a t+b
$$

where, the coefficient $a \cong k \omega_{0}$ and the instance $b \cong \varphi_{k}$ can be obtained from the fitting model. Therefore, the linear part of the total phase is retained by the linear regression. The carrier signal can be reconstructed as follows

$$
\hat{s}_{k}(t)=A_{k}(t) e^{i \widehat{\phi}_{k}(t)}
$$

This modulation signal of the bearing faults is composed of multiple components can be described by the individual mono-component signals. According to the Equation (6), the estimated coefficient $a \cong$ $k \omega_{0}$ is the $k$ th harmonic. The $\omega_{0}$ is the theoretical fault period of the bearing faults, which can be calculated using the theoretical fault frequency equations of the rolling element bearings. The variable $k$ can be estimated by

$$
k=\left\lceil\frac{a}{\omega_{0}}\right\rceil=\min \left\{m \in \mathbb{Z} \mid m \leq \frac{a}{\omega_{0}}\right\}
$$

The instantaneous phase of the lower and upper sidebands yields to

$$
\Phi_{k \pm j}(t)=(k \pm j) \omega_{0} t+\varphi_{k \pm j}+(k \pm j) n(t)
$$

The linearised instantaneous phase of the sidebands can be achieved by

$$
\widehat{\phi}_{k \pm j}(t)=\Phi_{k \pm j}(t)-\frac{(k \pm j)}{k}\left(\Phi_{k}(t)-\hat{\phi}_{k}(t)\right)
$$

Consequently, the lower and upper sidebands can be reconstructed as follows

$$
\hat{s}_{k \pm j}(t)=A_{k}(t) e^{i \widehat{\phi}_{k}(t)}
$$


In this manner the phase noise induced by random slippage within a bearing can be significantly supressed, allowing the reconstruction of the desired periodic signal.

Often in the case of incipient bearing faults, the original signals are severely contaminated by background noise, meaning that the estimate of the instantaneous phase inevitably introduces some noise into the reconstructed signals. After the phase correction, the cyclostationary signals are more approaching to theoretical periodic ones and then Equation (1) can more realistically represent the new phase relationship in the MSB. The new technique is named as the Phase Linearisation based Modulation Signal Bispectrum (PL-MSB), and the definition can be expressed as

$$
\widehat{B}_{M S}\left(f_{x}, f_{c}\right)=E\left\langle\hat{X}\left(f_{c}+f_{x}\right) \hat{X}\left(f_{c}-f_{x}\right) \hat{X}^{*}\left(f_{c}\right) \hat{X}^{*}\left(f_{c}\right)\right\rangle
$$

where, $\hat{X}(f)=\int_{-\infty}^{+\infty} \sum_{-j}^{j} \hat{s}_{k \pm j}(t) e^{-i 2 \pi f t} d t$ is the Fourier transform of the reconstructed signal. Figure 2 shows the basic steps of the proposed method and the details of each step are described in the following contents.

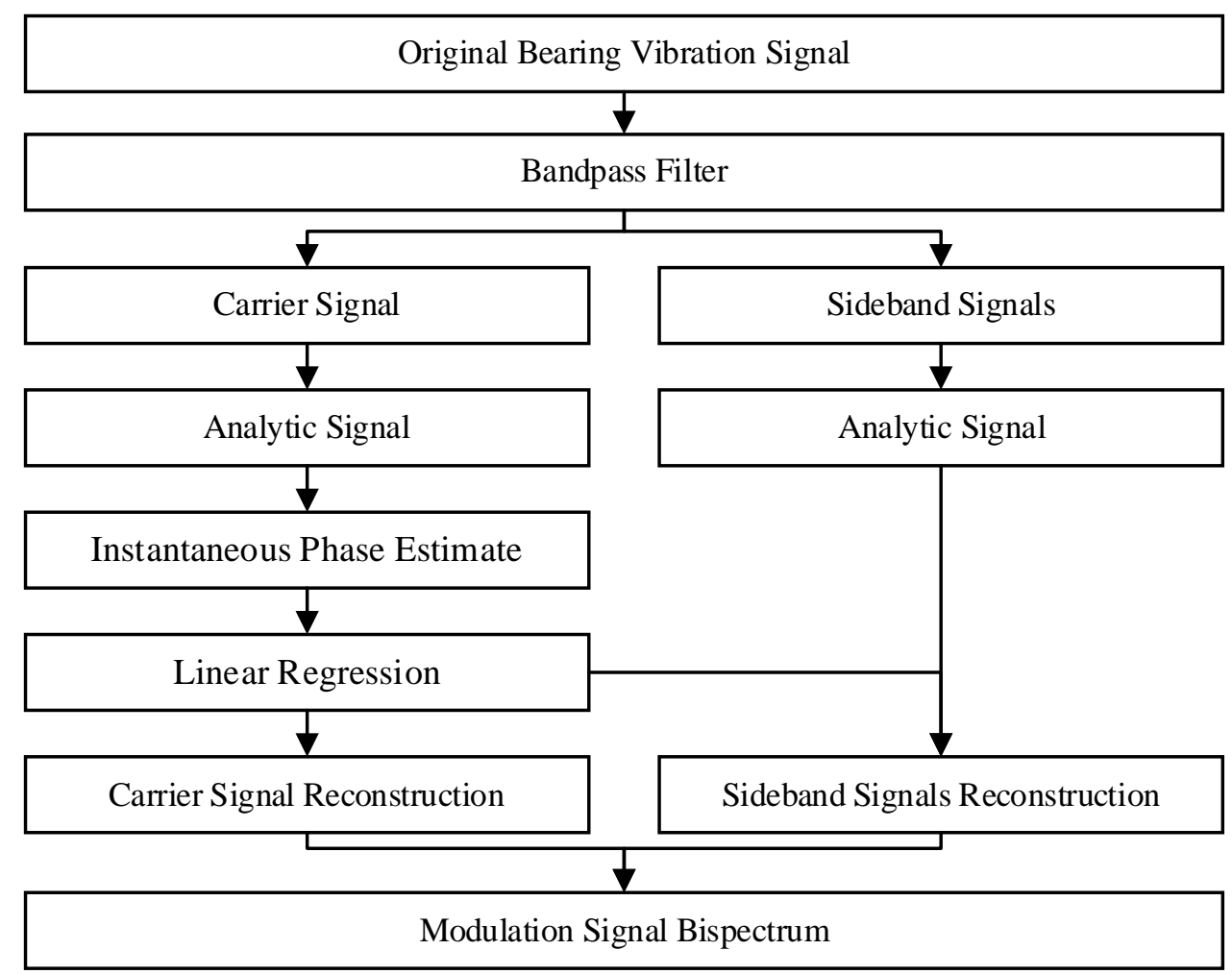

Figure 2 Flow chart of the PL-MSB method

The original vibration signals $s(t)$ are filtered to several narrow band signals, considered as the carrier signal and sideband signals, using the equally distributed and adjacent bandpass filters. The bandwidth of the bandpass filters is a bit larger than the target theoretical bearing fault frequency, of which the purpose is to include each harmonic of the bearing fault signal. The larger frequency bands are to ensure that the harmonic is located in the narrow frequency bands by considering the potential errors of the rotating speeds and geometric parameters. Totally seven bands are selected including one carrier $s_{k}(t)$, three lower sidebands $s_{k-1}(t), s_{k-2}(t), s_{k-3}(t)$ and three upper sidebands $s_{k+1}(t), s_{k+2}(t), s_{k+3}(t)$.

The filtered carrier signal $s_{k}(t)$ is then transformed by the Hilbert transform to obtain the analytic representation $s_{k}^{a}(t)$. The analytic signal retains all the information of the signal while discarding the negative frequency components. The absolute value of the analytic signal is the instantaneous amplitude or called the envelope. The inverse tangent function of the ratio between imaginary and real values is 
the instantaneous phase. The instantaneous phase $\widehat{\Phi}_{k}(t)$ of the filtered carrier $s_{k}(t)$ can be obtained from the analytic signals.

The linear regression model of the instantaneous phase $\widehat{\Phi}_{k}(t)$ is then developed to obtain the linear increment trend of the phase. The linear part $\hat{\phi}_{k}(t)$ is obtained to recover the periodicity of the narrow band carrier signal.

$$
\hat{s}_{k}(t)=\operatorname{Re}\left\{s_{k}^{a}(t) e^{i\left[\widehat{\phi}_{k}(t)-\widehat{\Phi}_{k}(t)\right]}\right\}
$$

Consequently the lower and upper sidebands can be reconstructed based on the linear part of the carrier phase. The analytic representations of the sidebands are obtained by the Hilbert transform and the reconstructed lower and upper sidebands can be obtained by the following equation

$$
\hat{s}_{k \pm j}(t)=R e\left\{s_{k \pm j}^{a}(t) e^{i\left[\frac{(k \pm j)}{k}\left(\widehat{\Phi}_{k}(t)-\widehat{\Phi}_{k}(t)\right)\right]}\right\}
$$

Then, a new signal with dominate deterministic parts can be obtained by the linear summation.

$$
\hat{s}(t)=\sum_{-j}^{j} \hat{s}_{k \pm j}(t)
$$

The reconstructed signal $\hat{s}(t)$ is more deterministic than the original signal $s(t)$ owing to the phase editing and correction. Thereafter the new signal can be effectively and efficiently used as the input of the Modulation Signal Bispectrum (MSB) to generate the MSB magnitudes and coherence for the early fault detection and diagnosis.

\section{Performance Evaluation by Simulation Study}

\subsection{Simulation of Bearing Signals}

A series of models was developed to simulate the vibration responses of bearings with localised defects 38-42. The fault related vibration responses from a rolling element bearing are induced by the periodic impulses when the rolling elements pass the localised fault. The system resonance modulates the periodic impulses, generating modulation vibration signals. In the reference ${ }^{38}$, bearing fault vibration is simulated by multiplying different vibration sources, for instance bearing induced vibration, nonuniform load, other machines induced vibration. However, this method is not an easy way to simulate in practical programming. In addition, the continuous frequency information in theoretical equations is nearly inevitable to be distorted in the simulated discrete signals. For the purpose of an easy but accurate way in practical simulations, a convolution method was developed to simulate the bearing modulation signals. Given that the rolling bearing is a linear system, the convolution operation between the successive impacts induced by rollers passing raceway defects and the impulse response function of the bearing system is used to generate bearing vibration signals, which can be expressed as follows.

$$
y(t)=h(t) * u(t)+w(t)
$$

where, $h(t)$ is the impulse system response of the bearing system; $u(t)$ denotes a series of periodic impacts when the rolling elements passing the defects. $w(t)$ represents the strong background Gaussian noise from other mechanical and electrical machines. The impulse response function of the bearing system is assumed to be a linear system with numerous modes, which can be shown as

$$
h(t)=\sum_{m=1}^{\infty} e^{-\eta_{m} 2 \pi f_{m} t_{h}} \cos \left(2 \pi f_{m} t_{h}\right)
$$

in which, $\eta_{m}$ is the damping factor of the resonant responses; $f_{m}$ is the natural frequencies of the bearing system at $m_{t h}$ order and $t_{h}$ is the vanish moment of the resonant responses. The periodic 
impacts by a localised fault in a purely rotating bearing can be represented using uniformly spaced pulses, which can be expressed as

$$
u(t)=\sum_{k=1}^{+\infty} A_{f}(t) \delta\left(t-k T_{f}\right)
$$

where, $A_{f}(t)$ is the impacts forces from the rolling element passing defects; $T_{f}$ is the characteristic period of bearing faults; $k$ denotes the $k_{t h}$ impacts during bearing rotations; and $\delta(\cdot)$ is the delta function, which is defined as

$$
\delta(t)= \begin{cases}1, & t=0 \\ 0, & t \neq 0\end{cases}
$$

If the outer race of a bearing is stationary and the inner race is rotating, the impact forces from outer race faults are constant due to the fixed location to the loaded zone. However, for a defect on the rotating inner ring, the impact forces will be modulated in amplitude by the shaft rotational frequencies, owing to the movement of the fault into and out of the loaded zone. Consequently, in the simulation fault impacts of the outer race and inner race faults are assigned as follows.

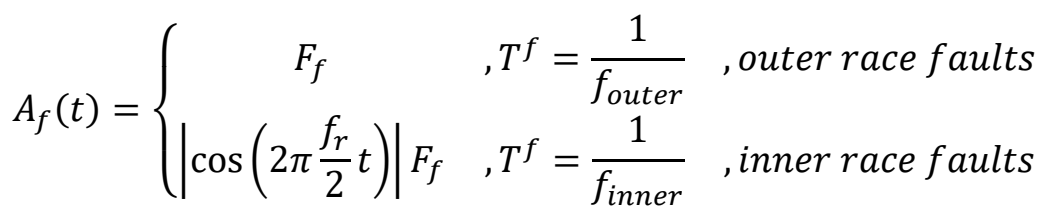

where, $F_{f}$ is the impact forces and $f_{r}$ is the shaft rotating frequency. In the simulation studies, the parameters of the noise-free vibration signal were set to typical fixed values as shown in Table 1.

Table 1 Key parameters of the simulated signals

\begin{tabular}{cc}
\hline Parameters & Value \\
\hline Sampling Frequency & $96000 \mathrm{~Hz}$ \\
Sampling Time & $90 \mathrm{~s}$ \\
Outer Race Fault Frequency & $89.8 \mathrm{~Hz}$ \\
Inner Race Fault Frequency & $135.2 \mathrm{~Hz}$ \\
Natural Frequencies & $2100 \mathrm{~Hz}, 5400 \mathrm{~Hz}$ and $11800 \mathrm{~Hz}$ \\
\hline
\end{tabular}

Figure 3 (a) shows the simulated bearing vibration signals by using Equation (26) and the consecutive transient responses are manifestly typical vibration signals of a rolling element bearing with outer race defects. Without slippage and speed variation, the transient responses occur periodically in theory. However, the rolling element moving into and out the loaded zones can lead to the random slippage between bearing components and consequently, results in the varying periods of the transient responses. The bearing signals are considered having cyclostationary characteristics, which degrades the performance of conventional MSB methods. To examine the proposed PL-MSB, five cases of bearing signals with five levels of slippage $(0,0.5 \%, 1 \%, 1.5 \%$ and $2 \%)$ were simulated to investigate the performance of the fault diagnosis, benchmarking by the milestone method of the conventional MSB. Figure 3 (b) depicts the variation of the fault periods in the simulated cases with the slippage of $0,1 \%$ and $2 \%$. 
(a) Impact Series

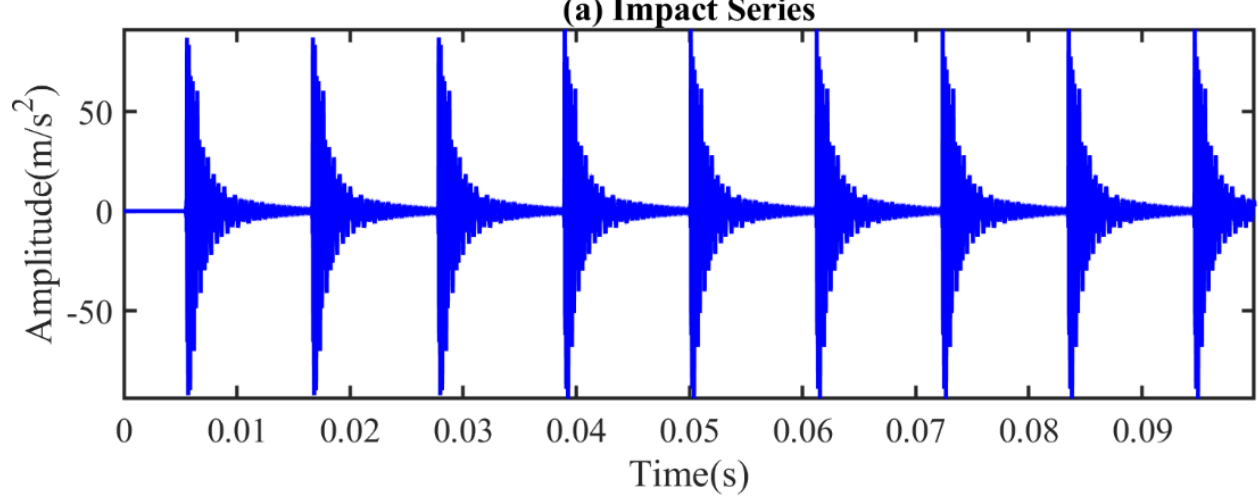

(b) Instantaneous Fault Frequency

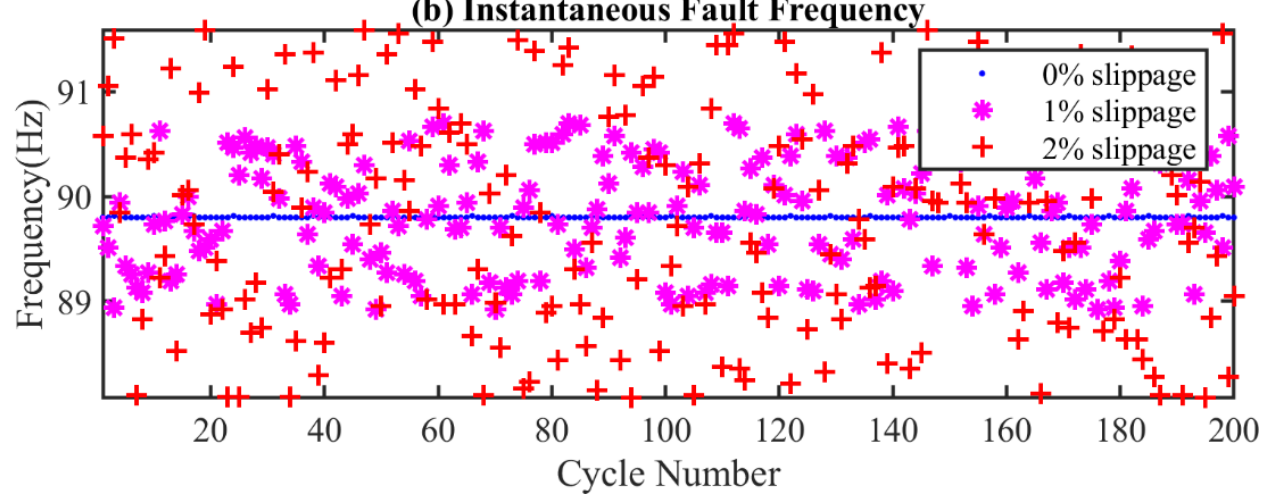

Figure 3 Simulated bearing outer race fault signals: (a) simulated bearing vibration with defects (b) fault periods varying due to slippage

The occurrence of slippages brings about random phase noise and the frequency components are not sparse any more, which is called the frequency smearing effect. As displayed in Figure 4, the bearing signal in the non-slippage case shows a sparse spectrum, which reveals the modulation effects induced by the periodic bearing impacts. However, in the other two slippage cases, the frequency components are blurred and compose a continuous spectrum in the frequency domain. Moreover, the higher level of the random slippage, the more severe smearing effects of the signals. It also can be seen that the high frequency components are susceptible to the bearing slippage and vice versa. The random slippage brings the phase noise in the time domain, which makes the theoretically pure amplitude modulation signal into a nonlinear combination of the amplitude modulation signal and phase modulation signal.

According to the spectra of the simulated signals, the random slippage can distort the periodic signals and results in a significantly decrease of more than $70 \%$ for the amplitude around the natural frequencies in the 4 cases of the slippage simulation.

In the Figure 5 (a), the extremely high level of white noise was added to the bearing signal with $2 \%$ random slippage, of which the SNR is $-22 \mathrm{~dB}$. The transient responses are completely submerged by the background noise in the time domain. As a result, the bearing responses are entirely shielded by the noise in the frequency domain. Figure 5 (b) and (c) show the flat spectrum dominated by the white Gaussian noise. The non-slippage signal has a higher amplitude, which allows the bandpass filter to improve the signal quality and hence achieves the successful fault detection and diagnosis. The signal with random slippages is attenuated by the phase noise in the frequency domain and the useful information is fully covers by the noise. Identification of the bearing faults in the cyclostationary signals is a changeling subject, especially for the incipient bearing fault. 


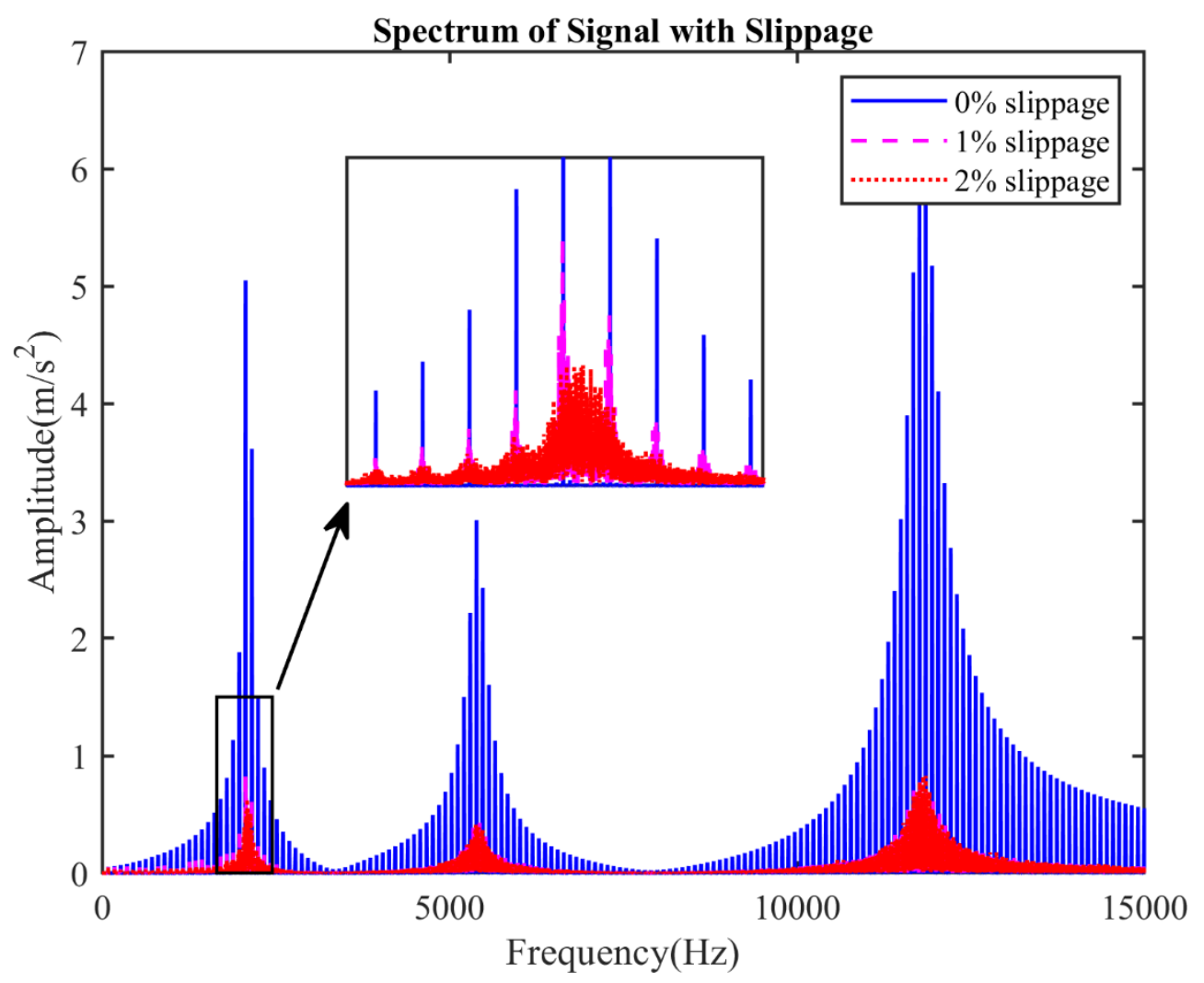

Figure 4 Amplitude spectra of simulated bearing outer race fault signals at different slippages

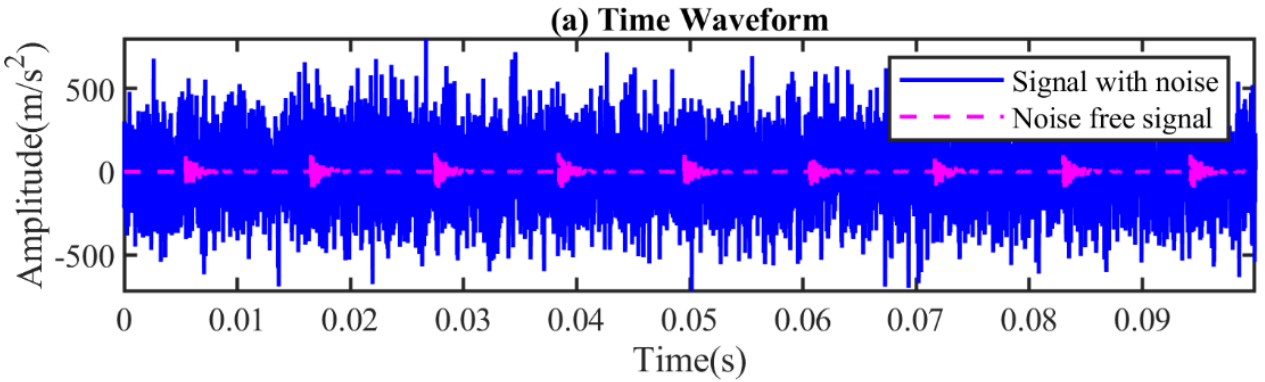

(b) Spectrum

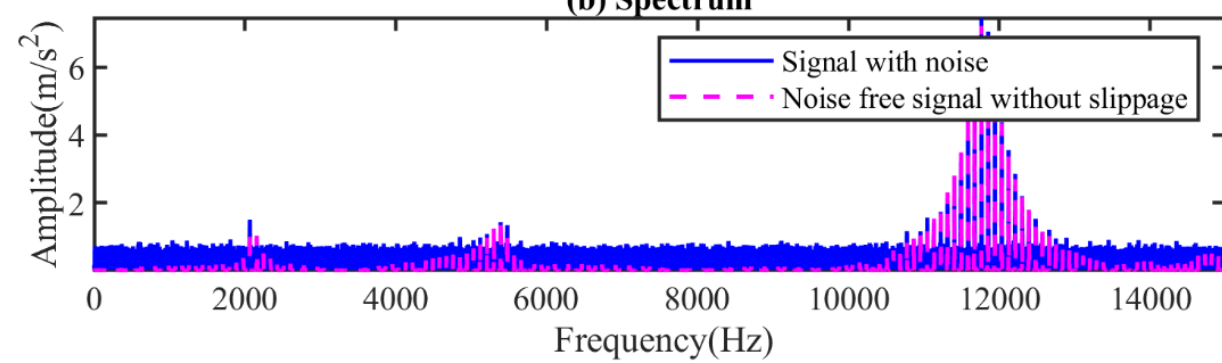

(c) Spectrum

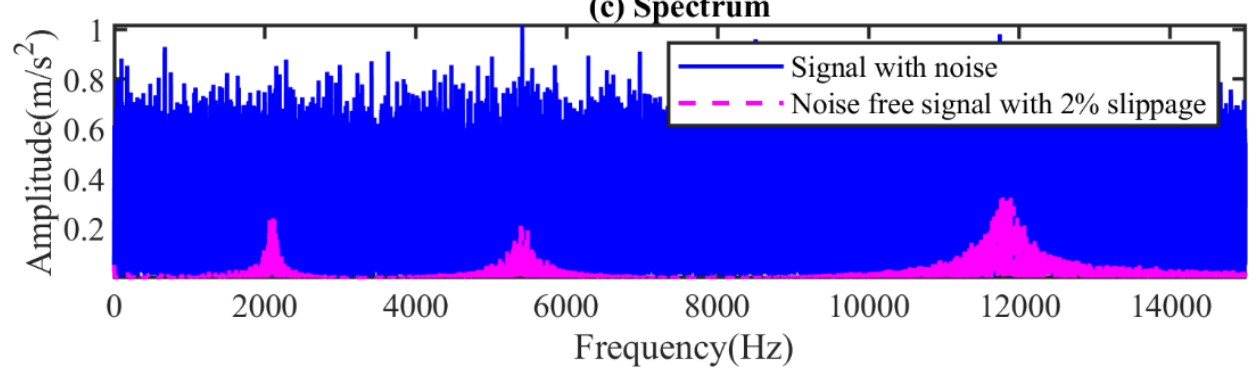

Figure 5 Simulated bearing outer race fault signals at the SNR of -22dB: (a) time waveforms with $2 \%$ slippage,

(b) spectra of the signal without slippage, (c) spectra of the signal with $2 \%$ slippage 


\subsection{Simulation Study of PL-MSB}

The bearing signals at different levels of slippage were simulated and then, the proposed method is examined by these simulated signals. To demonstrate the capability of the developed method, the following analysis is based on the worst case with $2 \%$ random slippage at the SNR of $-22 \mathrm{~dB}$. Figure 6 shows the spectrums of the original and phase linearised signals respectively. To reduce the interference of other components and noise, a narrow bandpass filter is manually selected from $11753.6 \mathrm{~Hz}$ to $11846.4 \mathrm{~Hz}$, which is centred at the third natural frequency $11800 \mathrm{~Hz}$ with a bandwidth of $92.8 \mathrm{~Hz}$ (the outer race fault frequency of $89.8 \mathrm{~Hz}$ plus an extra tolerance of $3 \mathrm{~Hz}$ ). The instantaneous phase estimated from the analytic signal is linearised by the proposed method to eliminate the undesired phase noise. Three lower and upper sidebands are also filtered by six bandpass filters. The frequency bands are listed in the Table 2.

Table 2 Bandpass filters

\begin{tabular}{cc}
\multicolumn{2}{c}{ Table 2 Bandpass filters } \\
\hline Carrier signal & {$[11753.6 \mathrm{~Hz}, 11846.4 \mathrm{~Hz}]$} \\
Lower sidebands 1 & {$[11660.8 \mathrm{~Hz}, 11753.6 \mathrm{~Hz}]$} \\
Lower sidebands 2 & {$[11568.0 \mathrm{~Hz}, 11660.8 \mathrm{~Hz}]$} \\
Lower sidebands 3 & {$[11475.2 \mathrm{~Hz}, 11568.0 \mathrm{~Hz}]$} \\
Upper sidebands 1 & {$[11846.4 \mathrm{~Hz}, 11939.2 \mathrm{~Hz}]$} \\
Upper sidebands 2 & {$[11939.2 \mathrm{~Hz}, 12032.0 \mathrm{~Hz}]$} \\
Upper sidebands 3 & {$[12032.0 \mathrm{~Hz}, 12124.8 \mathrm{~Hz}]$} \\
\hline
\end{tabular}

Consequently, the summation of the reconstructed carrier and six sidebands leads to a new signal. The deterministically periodic components become principal of the reconstructed signals. As shown in Figure 6, the linearised phase method can effectively rectify the original signal and thus, lead to a sparse spectrum in the frequency domain. The linear process retains the periodic signals and eliminates the phase noise, which can produce a new signal with ample SNR and allow MSB to extract the fault features efficiently and effectively.

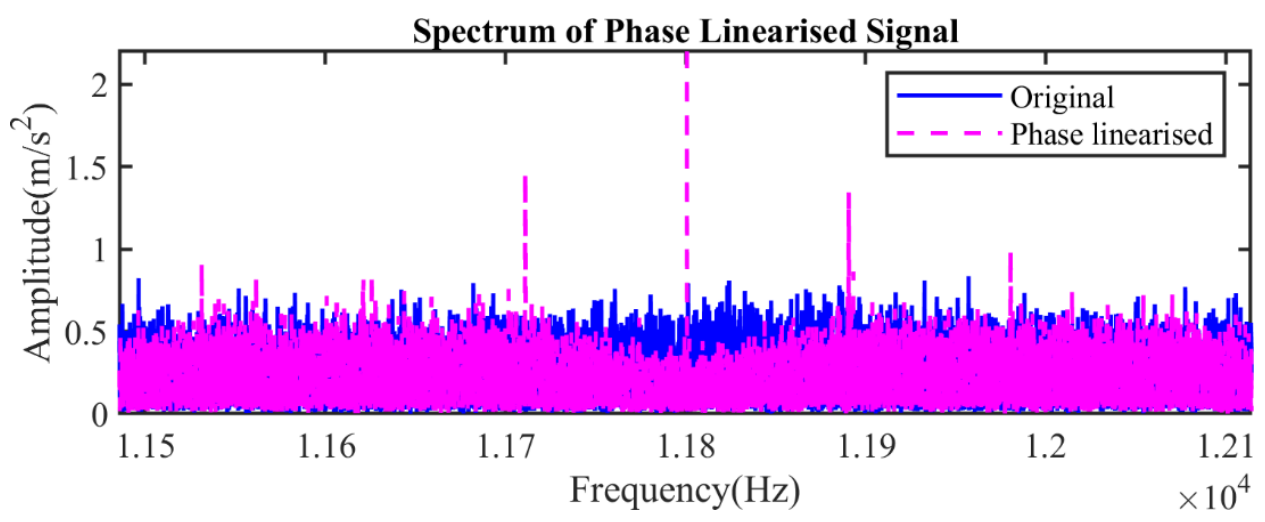

Figure 6 Phase linearised outer race fault signals at 2\% slippage and SNR-22dB

Figure 7 shows the final results of the PL-MSB. The bispectrum of the MSB are displayed in Figure 7 (a) and it presents a clean and sparse bispectrum of the modulation signal. The fault frequency of the outer race fault as well as the harmonics can be seen clearly in the PL-MSB. The corresponding coherence in the Figure 7 (b) denotes that the extracted fault signatures in the PL-MSB can be further verified by the coherence because of normalisation. In this outer race fault case, the fault frequency is $89.8 \mathrm{~Hz}$ and the carrier frequency is $11800 \mathrm{~Hz}$. The sparse high coherence value points at $(89.8,11800)$, $(89.8 \times 2,11800)$, and $(89.8 \times 3,11800)$ denote that the corresponding features in the PL-MSB spectrum are extracted with high SNR. Besides the main peaks, the other peaks at $(89.8,11800 \pm 89.8)$ and $(89.8 \times 2,11800 \pm 89.8)$ also show the obvious coupling relationship, which is the modulation 
phenomenon in bearing vibration signals. The high coherence values show that the extracted fault features have high coupling relationship and high SNR after the sufficient averaging.

(a) PL-MSB

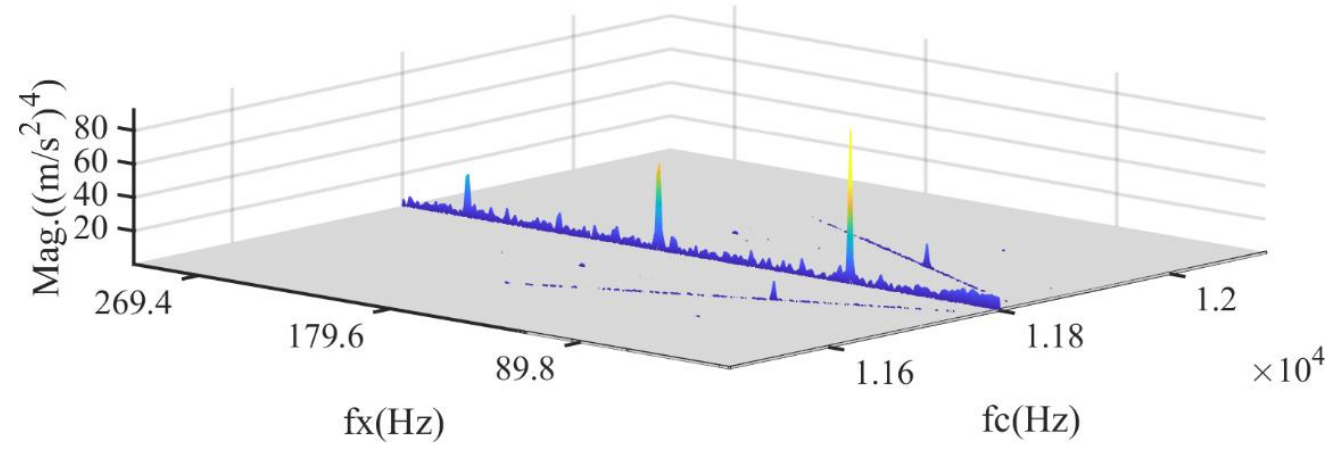

(b) PL-MSB Coherence

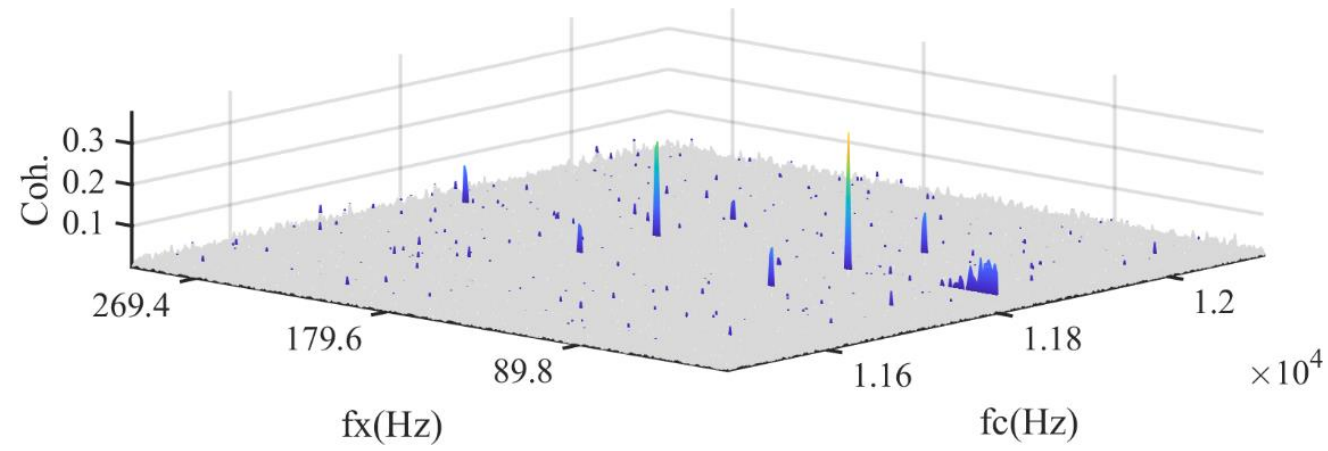

Figure 7 PL-MSB of outer race faults at 2\% slippage and SNR-22dB: (a) bispectrum (b) coherence

Figure 8 displays the spectrum and the coherence of the conventional MSB and it shows that the benchmark method is difficult to extract the fault signatures due to the contamination of the phase noise.

(a) MSB

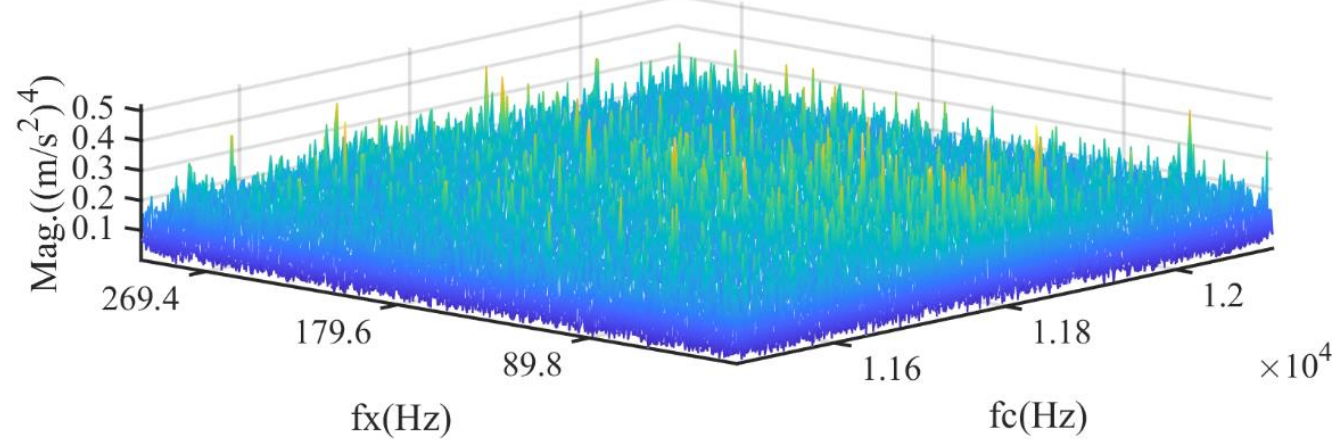

(b) MSB Coherence

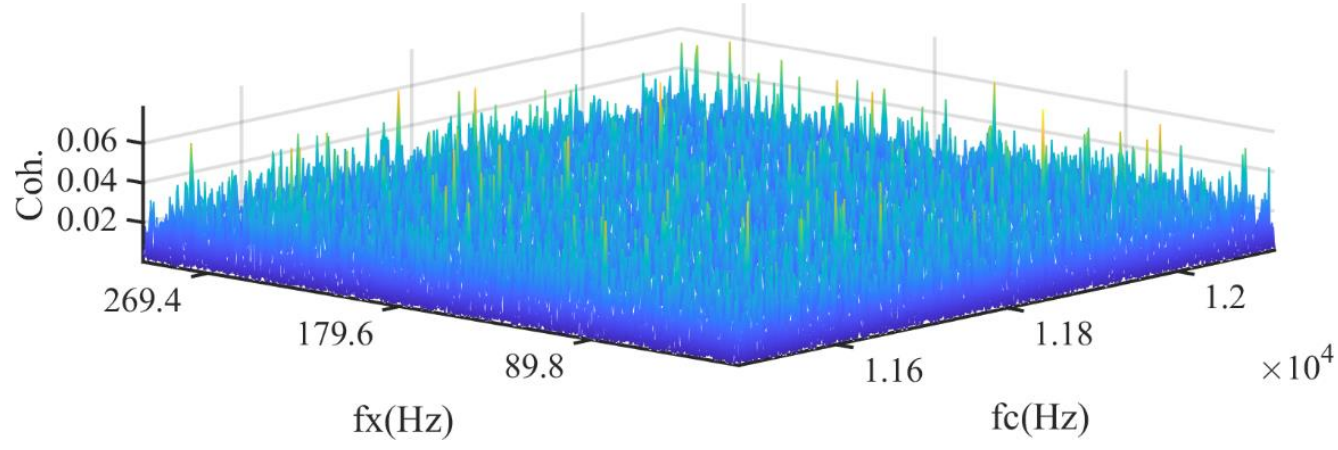

Figure 8 Conventional MSB of outer race faults at 2\% slippage and SNR-22dB: (a) bispectrum (b) coherence 
The PL-MSB and MSB generate a large matrix representation over the $\left(f_{x}, f_{c}\right)$ plane. As a certain degree of the expertise is required to understanding the three dimensional results, a more concise illustration, MSB Slice, is used to make the high order spectrum more interpretable and comparable, of which the selection can be expressed as

$$
S\left(f_{x}\right)=\left|\hat{B}_{M S}\left(:, f_{c}\right)\right|_{\exists f_{c}: \max \left(\hat{B}_{M S}\left(\min \left(f_{x}\right), f_{c}\right)\right)}
$$

where, $\exists f_{c}: \max \left(\widehat{B}_{M S}\left(\min \left(f_{x}\right), f_{c}\right)\right)$ denotes the way to selecting the MSB Slice. The rows of MSB matrix is corresponding $f_{x}$ and the columns of MSB matrix is corresponding $f_{c}$. The selection of the MSB Slice is to find the maximum value in the first row $\left(\right.$ at $\left.\min \left(f_{x}\right)\right)$ of the MSB matrix. The maximum value is corresponding to the most pronounced carrier frequency $f_{c}$. To make the results more concise and visualized, the MSB results are represented by the MSB slices in the following comparable studies.

Figure 9 (a) and (b) show the MSB slices of PL-MSB and MSB respectively. These results are from the cases with $2 \%$ slippages at SNR of $-22 \mathrm{~dB}$. The results illustrate that the PL-MSB is robust to the random slippage induced phase noise. The conventional MSB is an outstanding method to suppress the random noise and decouple the modulation signals, but it suffers from the notorious distortion of the phase noise. As depicted in Figure 10 (b), the MSB slice cannot show the fault signatures in the simulated signal, which is difficult to give a promising result in such conditions. In contrast, the PL-MSB slice demonstrates that the PL-MSB can effectively supress the phase noise as well as the background noise, and hence, lead to the accurate diagnosis of the bearing faults in early stages.

(a) PL-MSB Slice

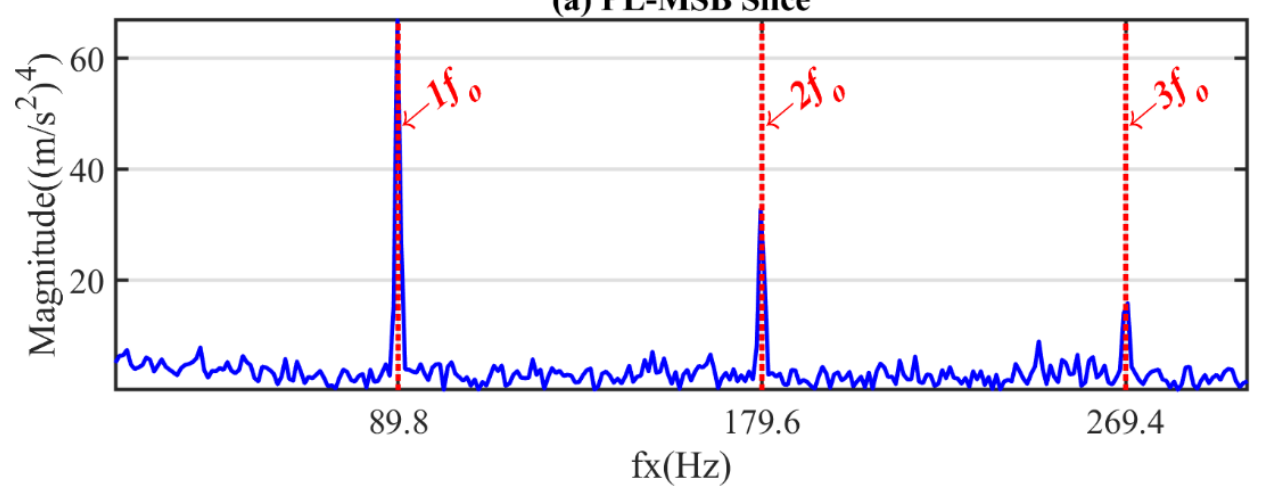

(b) MSB Slice

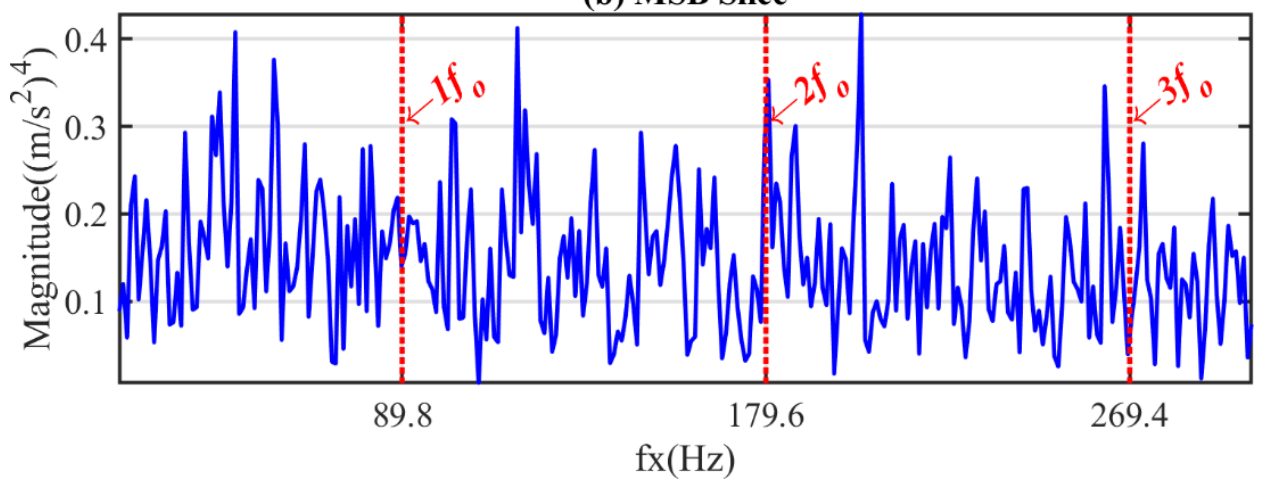

Figure 9 Slice of bispectrum for the outer race fault at 2\% slippage and SNR -22dB: (a)PL-MSB slice (b) MSB slice

The diagnostic results of the five slippage cases for the bearing outer race fault are shown in the Figure 10. The PL-MSB slices of the five slippage cases at the SNR of -22dB show that the PL-MSB can generate promising detection and diagnosis for the outer race faults at different levels of phase noise and severe background noise. The conventional MSB can only diagnose the outer race faults in the circumstance of no slippage. For the other four slippage cases, the conventional MSB cannot effectively 
characterise the cyclostationary signals due to the limited ability of the Fourier transform upon the nonstationary signals.

(a) PL-MSB Slice

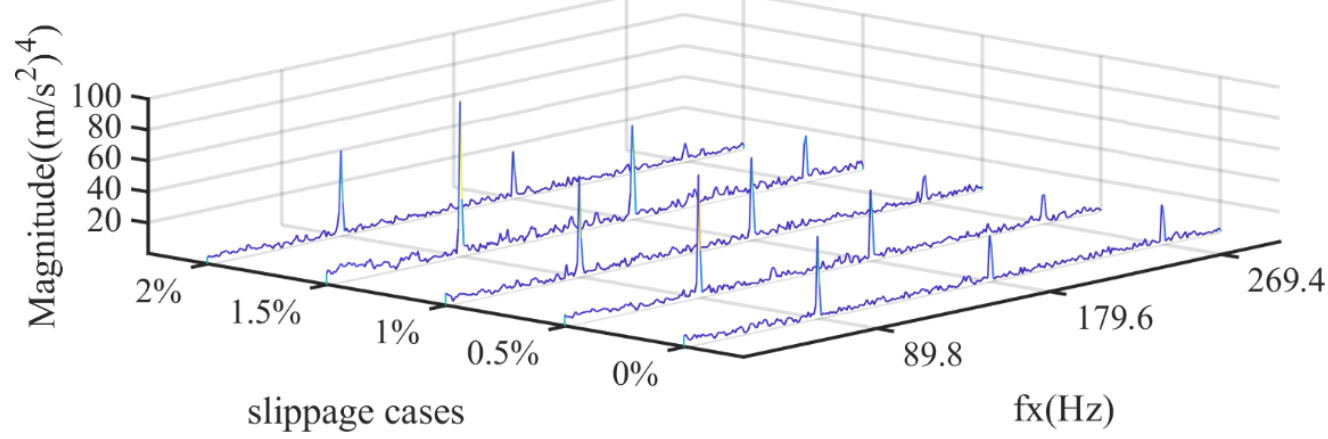

(b) MSB Slice

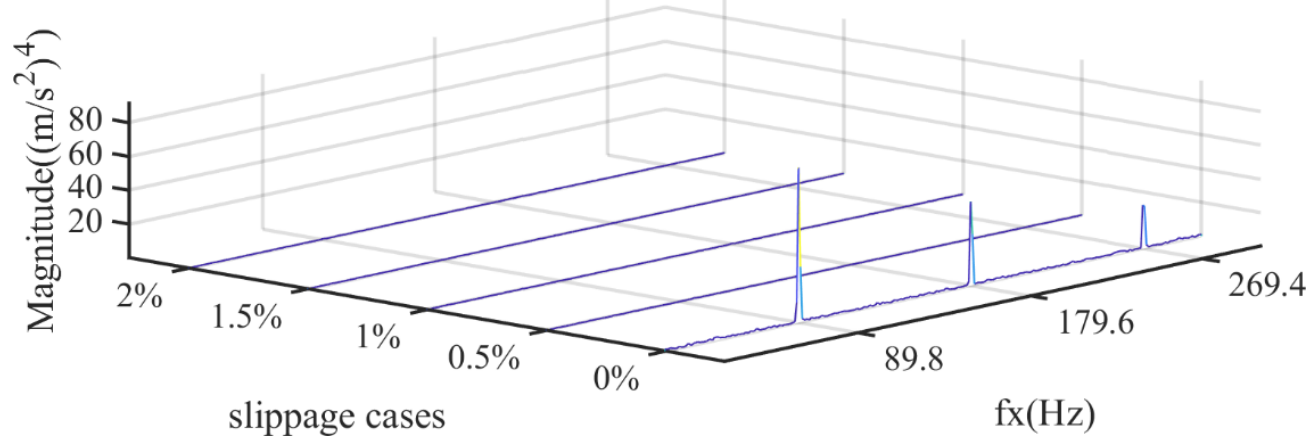

Figure 10 Diagnostic results of outer race faults at SNR-22dB: (a) PL-MSB slices (b) MSB slices

For the inner race fault cases, the same processing steps are used to detect and diagnose the inner face defects. Figure 11 (a) depicts that the PL-MSB can extract the inner race fault information at the case of $2 \%$ slippage and SNR -22dB. Furthermore, the sidebands around the fault frequency of the inner race is also manifest. The conventional MSB in Figure 11 (b) fails to detect the bearing inner race faults.

Figure 12 gives the diagnosis results of the two methods for the five inner race fault cases. The PLMSB slices in the Figure 12 (a) show that the proposed method is robust and accurate to the phase noise and background noise and the PL-MSB successfully achieves the effective detection and diagnosis of the bearing inner race faults. In contrast, the conventional MSB cannot identify the bearing faults for all the slippage cases. 
(a) PL-MSB Slice

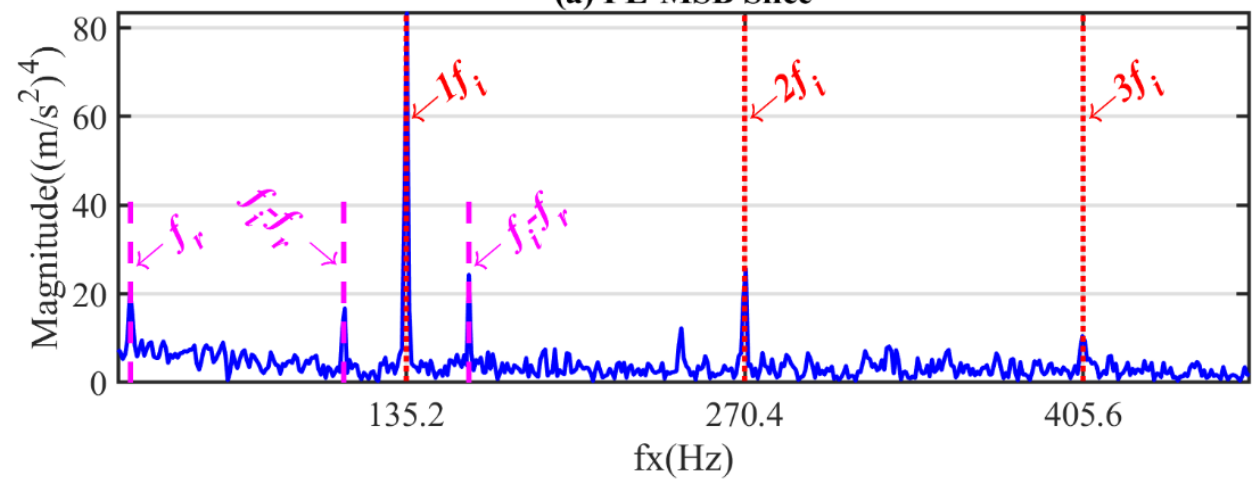

(b) MSB Slice

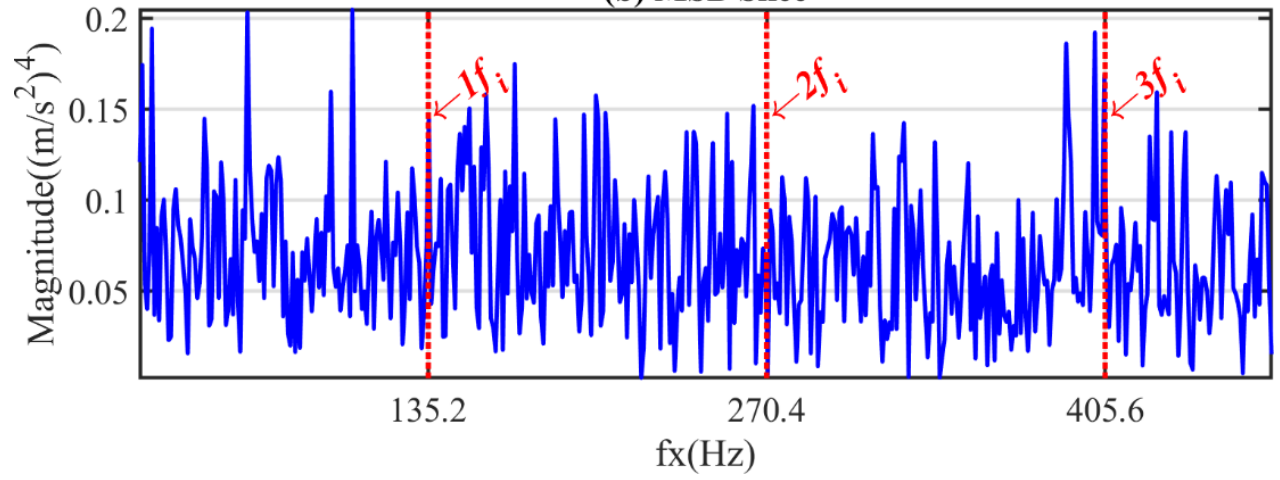

Figure 11 Slice of bispectrum for the inner race fault at 2\% slippage and SNR -22dB: (a)PL-MSB slice (b) MSB slice

(a) PL-MSB Slice

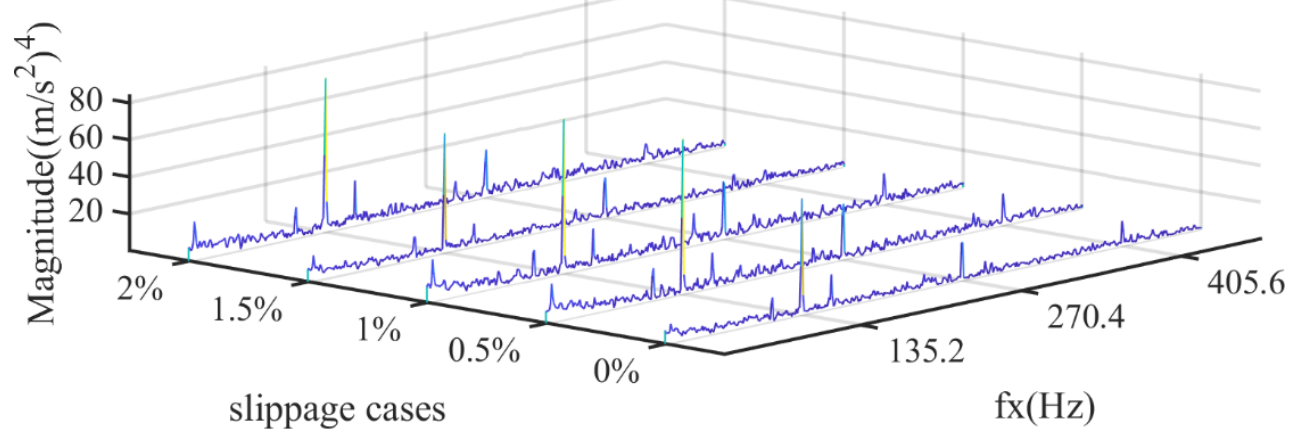

(b) MSB Slice

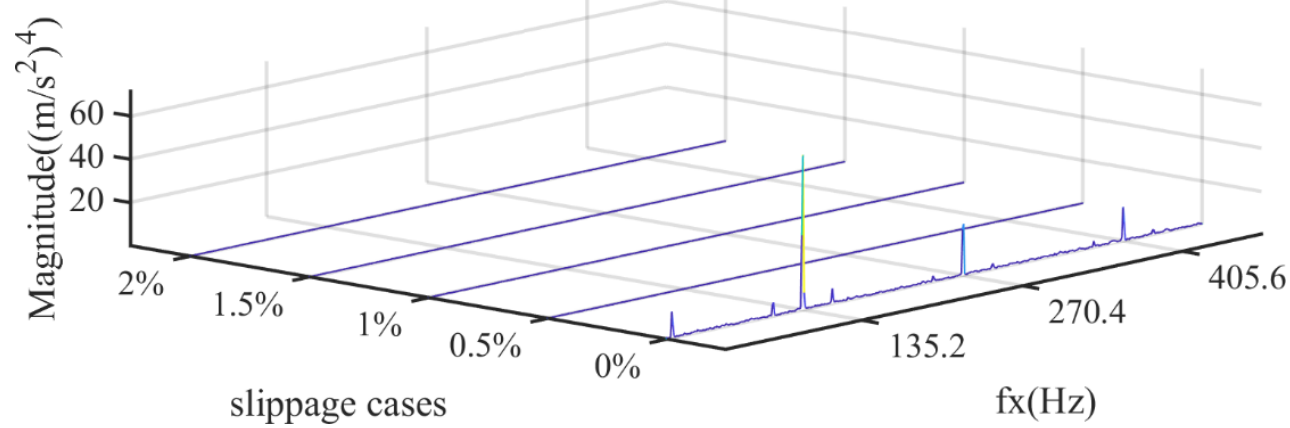

Figure 12 Diagnostic results of inner race faults at SNR-22dB: (a) PL-MSB slices (b) MSB slices 


\section{Experimental Evaluation}

\subsection{Bearing Fault Simulation and Tests}

To further verify the proposed approach of the Phase Linearisation based Modulation Signal Bispectrum, the experimental study was conducted using an in-house bearing test system. The common rolling bearings are hardly possible to control the working conditions at a certain degree of random slippage. Rather than using the conventional radial roller bearing, this experimental study employed a tapered roller bearing to achieve the controllable both the extent of slippage and the SNR. The tapered bearing based test rig is hence straightforward to produce the highly challenging signals for examining the capability of the proposed fault detector.

Figure 13 shows the layout of the tapered bearing based test rig. The main shaft is supported by the tapered bearing and a double deep groove ball bearing. Connected by the flexible coupling, the shaft is driven by a four pole induction motor. As shown in Figure 13 (b), the outer race of the test bearing was fixed within a housing and the clearance of the test bearing was adjusted by the movement of an adjusting nut through the threads on the shaft. It is clear that the clearance between bearing components directly influence the contact stiffness, which dominates the rotating conditions of the rollers. Consequently, the different preload can be exerted on the tapered bearing for achieving the various extent of random slippage.

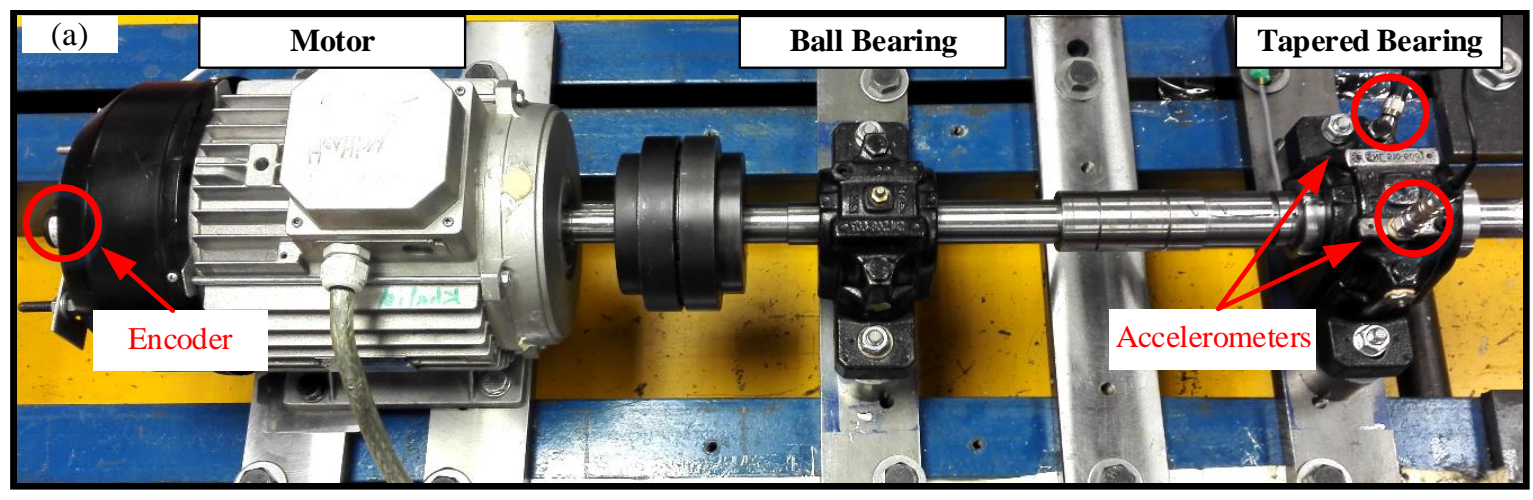

(b)

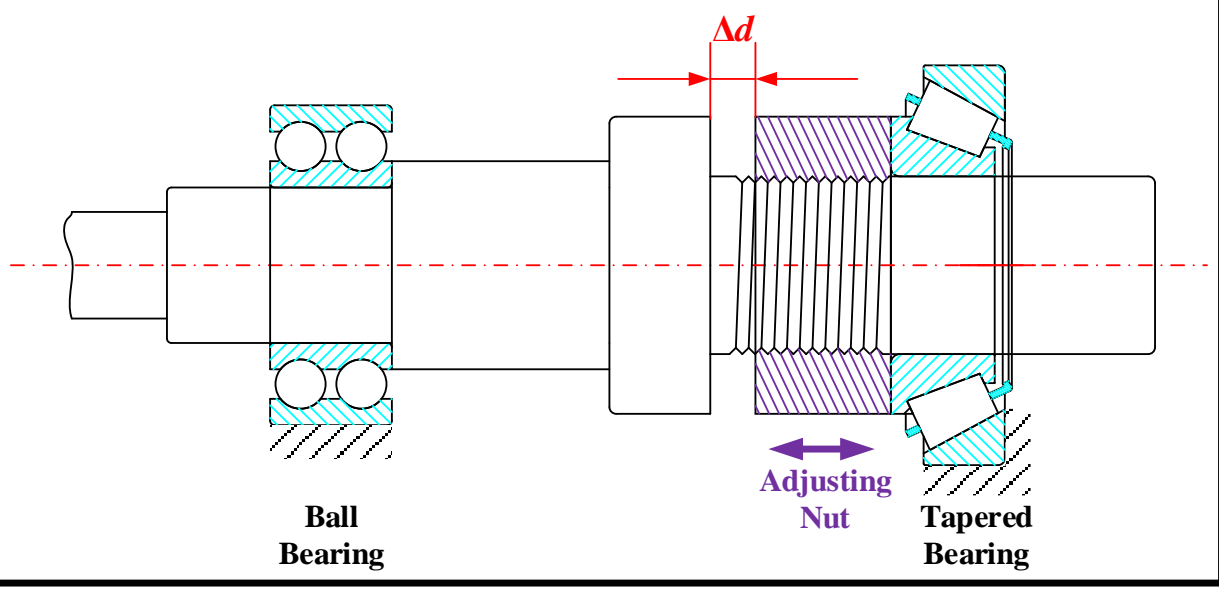

Figure 13 Test rig for the tapered roller bearing: (a) photograph, and (b) schematic diagram

Two accelerometers and a thermocouple were installed on the tapered bearing housing to measure vibration and temperature of the bearing with different clearance. An encoder was installed on the fan end of the induction motor to monitor the rotating speed. Key specifications of the data acquisition system and the target bearing are listed in Table 3. 
Table 3 Specifications of the data acquisition system

\begin{tabular}{lll}
\hline Item & Model & Key Specifications \\
\hline Date Acquisition Card & NI 6221 & $16-\mathrm{bit}, 250 \mathrm{kS} / \mathrm{s}$ overall \\
Charge Amplifier & B\&K 2635 & $0.2 \mathrm{~Hz}-100 \mathrm{kHz}$ with noise $<5 \times 10^{-3} \mathrm{pC}$ \\
Accelerometers & CA-YD-104T & $3.640 \mathrm{pC} / \mathrm{ms}^{-2}$ in $0.5 \mathrm{~Hz}-7000 \mathrm{~Hz}$ \\
Thermocouple & K type & $0-200^{\circ} \mathrm{C}$ range, $\pm 1.1^{\circ} \mathrm{C}$ \\
Encoder & RI32 & $100 \mathrm{ppr}, \max$ speed $6000 \mathrm{rpm}$ \\
Tapered Bearing & 31308 & $40 \mathrm{~mm}, 90 \mathrm{~mm}, 25.25 \mathrm{~mm}$ \\
\hline
\end{tabular}

Figure 14 shows a line scratch with length $3 \mathrm{~mm}$, width $0.1 \mathrm{~mm}$ and depth $0.05 \mathrm{~mm}$ was seeded on the outer race of the tapered bearing. This tiny fault was used to simulate the bearing defect in a very early stage. Moreover, only half of the scratch length can be contacted with the rolling elements during the rotating of the bearing, which only accounts for about $11 \%$ of the roller length.
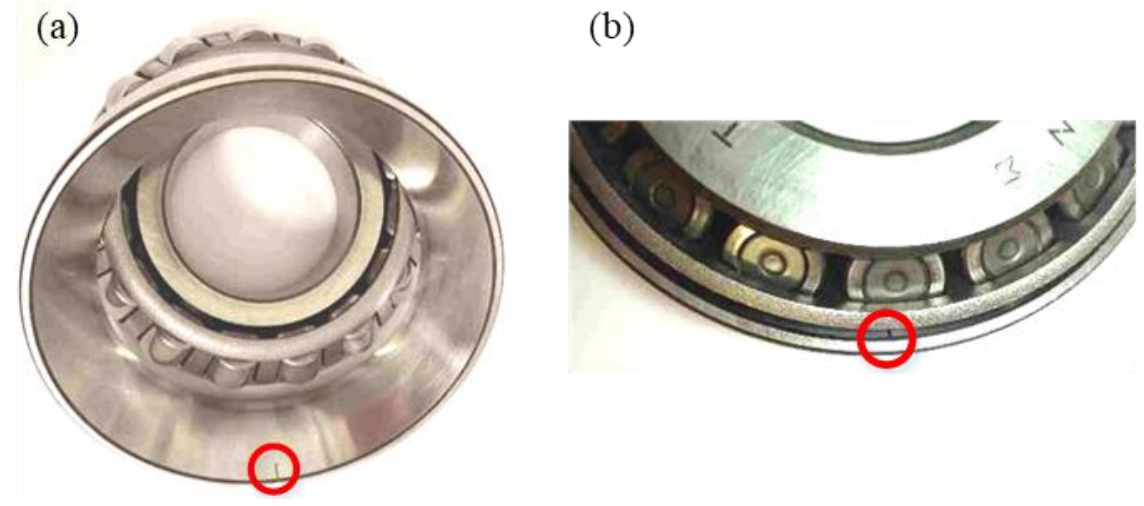

Figure 14 Photograph of the outer race fault of the bearing: (a) with bearing disassembled, and (b) with bearing assembled

Three levels of internal clearances were operated to acquire the vibration signals by adjusting the nut in the axial direction. The greater the gap $\Delta d$ between the adjusting nut and the shaft step is, the smaller the internal clearance between the bearing components becomes. As detailed in Table 4, these clearance levels (Zero, Medium and Large) correspond to wear degrees of Small, Medium and Large respectively. At each level, the test rig is controlled to run at $100 \%$ speed--1500rpm via variable frequency drive-for 5 minutes and the data acquisition system starts to log data from the third minute at the stable stage. All channel signals were sampled simultaneously for 30 seconds using the high-speed and 16-bit data acquisition system at a sample rate of $50 \mathrm{kHz}$.

Table 4 Test cases

\begin{tabular}{lll}
\hline Test Cases & Speed & $\boldsymbol{\Delta} \boldsymbol{d}$ \\
\hline Zero Clearance & $100 \%$ & $14.85 \mathrm{~mm}$ \\
Medium Clearance & $(1500 \mathrm{rpm})$ & $14.81 \mathrm{~mm}$ \\
Large Clearance & & $14.77 \mathrm{~mm}$ \\
\hline
\end{tabular}

\subsection{Evaluation of PL-MSB}

Figure 15 (a) shows the acquired vibration signals from the vertical accelerometer on the housing of the tapered roller bearing. In the time domain, the defect induced impulse are barely distinguishable, which means the collisions between the rollers and the outer race are weak. In such poor condition, the classic band selection methods including Fast Kurtogram are no longer effective. Instead of using these methods, the power spectral density (PSD) is more reliable by selecting the peaks in the frequency domain because the natural frequencies play the carrier role in bearing vibration signals and usually the spectrum in resonant zones have a pronounced amplitude. Therefore, the power spectrum density (PSD) 
is recommended to assist the manual selection of the optimal frequency bands. As shown in Figure 15 (b), the PSD function is estimated by the Welch's method and three frequency bands are manually selected based on the amplitude in PSD.

(a) Vibration of Tapered Bearing at Zero Clearance

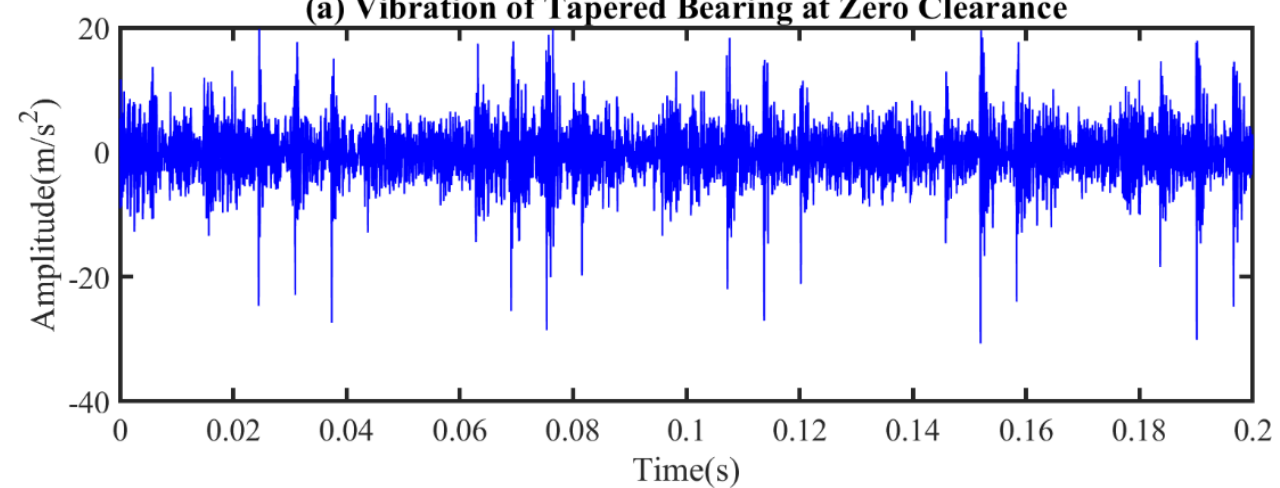

(b) Vibration Spectrum of Tapered Bearing at Zero Clearance

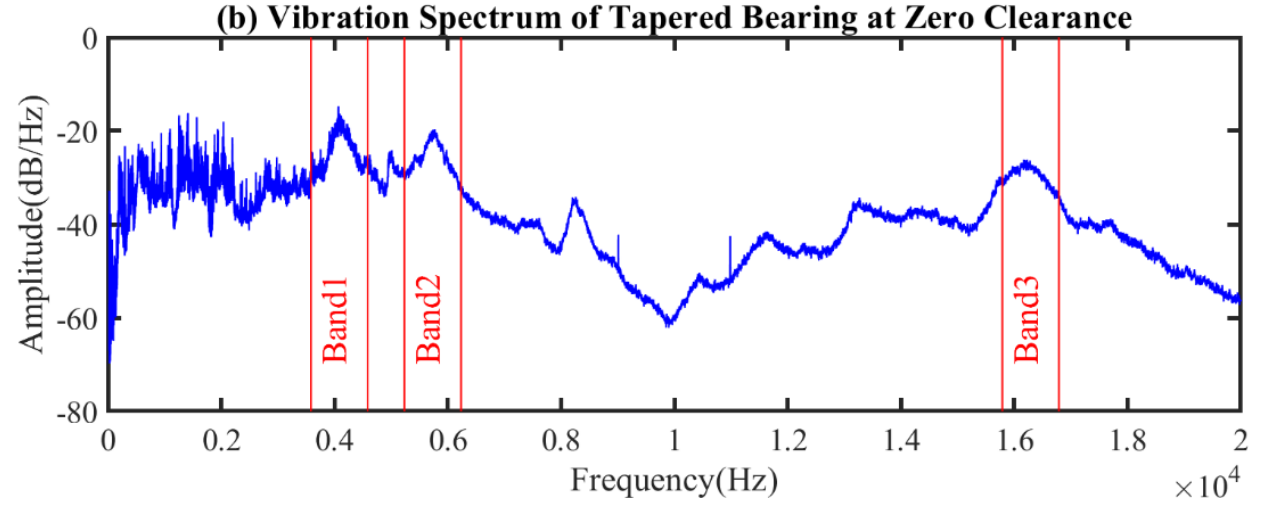

Figure 15 Vibration signals of the tapered bearing: (a) time domain signal of the tapered bearing at zero clearance, and (b) power spectrum density of (a)

After the trial studies, the diagnosis results based on Band 1 are the best and hence the diagnostic results of PL-MSB and MSB are given based on the Band 1 from $3525.35 \mathrm{~Hz}$ to $4644.65 \mathrm{~Hz}$ with the bandwidth of 7 times of the theoretical fault frequency of the outer race defect at the rotating speeds of 1500rpm. Figure 16 and Figure 17 shows the PL-MSB and MSB of the bearing vibration signals at the large clearance respectively. The bispectrum and bicoherence of the PL-MSB in the Figure 16 show that the phase linearisation makes the bearing vibration signals more stationary and can be effectively characterised by the MSB. The fault signatures show that the proposed method can detect the outer race defect with high accuracy. The coherence of the PL-MSB can reveal that the low amplitude components associated with faults in the bispectrum can be found clearly in the bicoherence owing to the normalisation. The sidebands around the fault frequency is usually not visible in the cases of the outer race failure. The appearance of the rotating frequency sidebands is due to the imbalance of the shaft. The fundamental frequency of the outer race defect as well as the $2^{\text {nd }}$ and $3^{\text {rd }}$ harmonics demonstrate the fault occurrence with high confidence. Moreover the noise floor in the spectrum is very low owing to the outstanding noise reduction in the phase and amplitude perspectives of the proposed PL-MSB. The conventional MSB in Figure 17 shows that the vibration signals from the tapered roller bearings are highly nonstationary and the conventional method is not valid. The bispectrum of MSB is difficult to reveal the exist faults in the bearings. The bicoherence with a very small amplitude further tells that the desired information is submerged into the phase noise and background noise. 
(a) PL-MSB

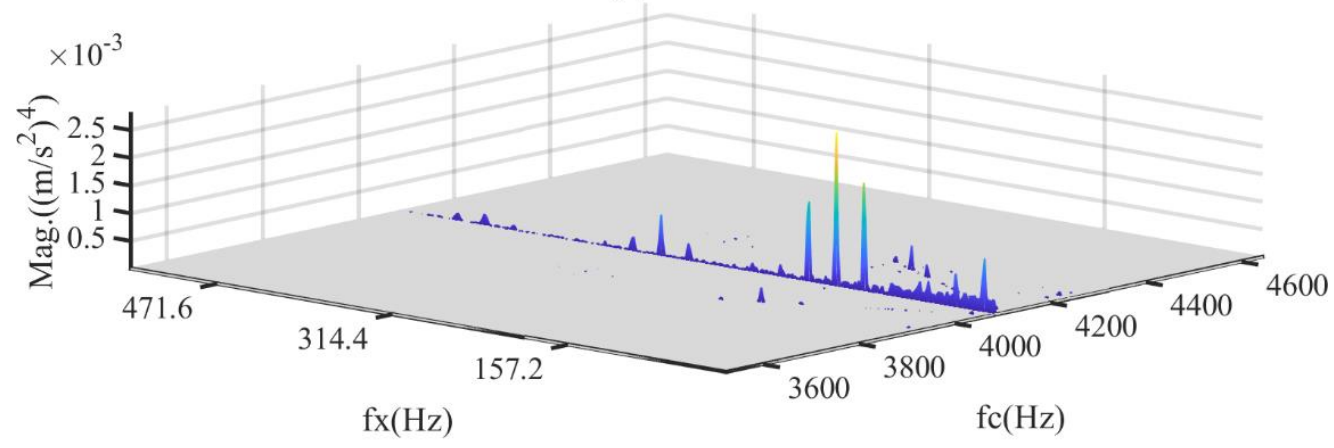

(b) PL-MSB Coherence

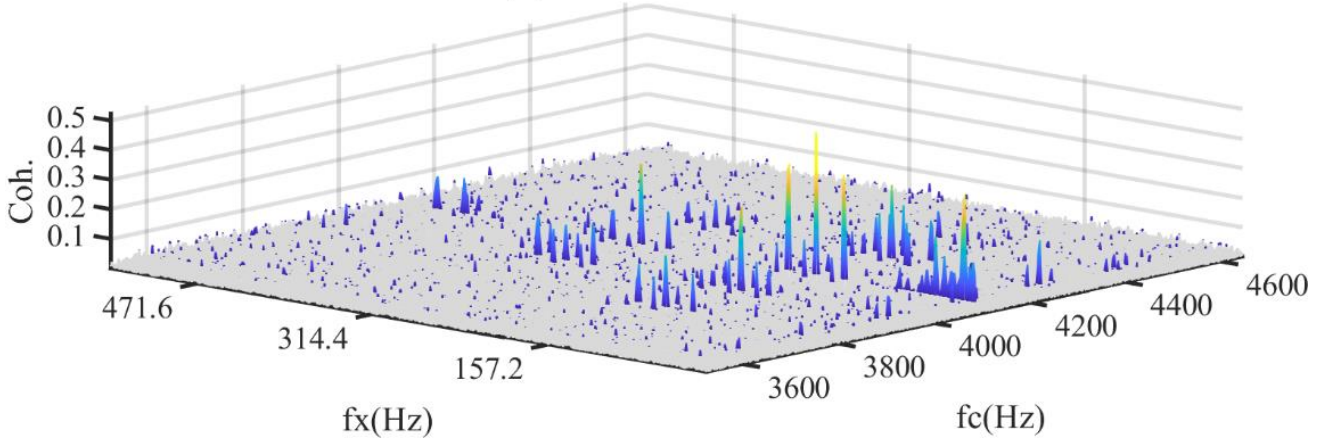

Figure 16 PL-MSB of outer race faults at large clearance: (a) bispectrum (b) coherence

(a) MSB

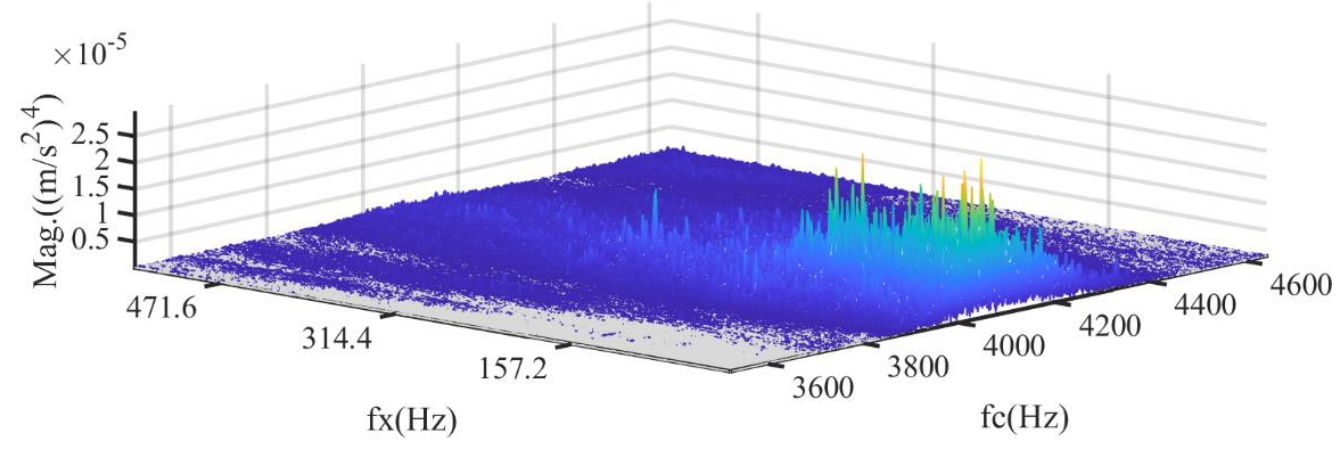

(b) MSB Coherence

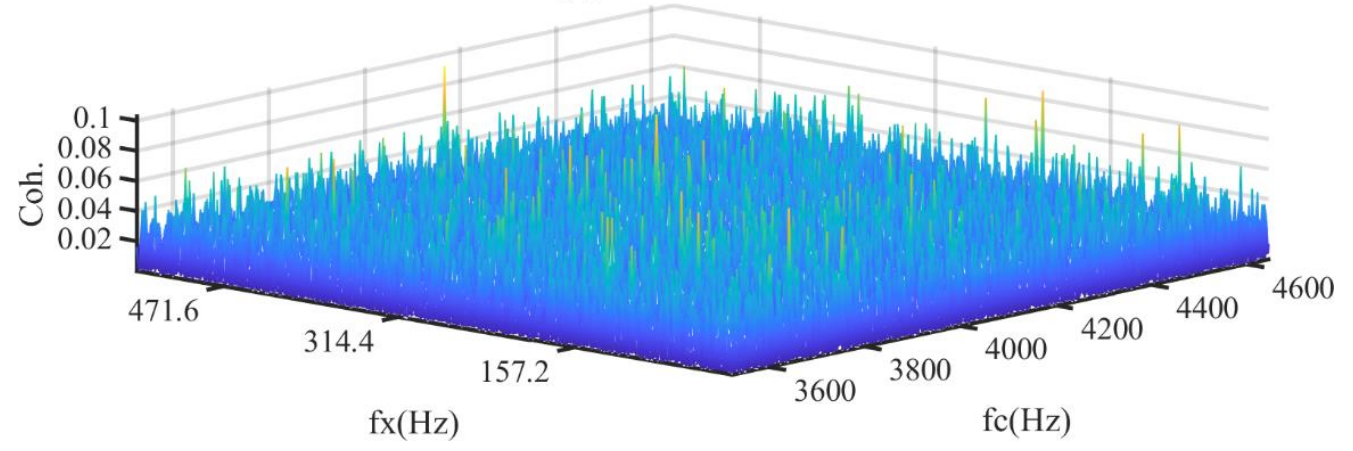

Figure 17 Conventional MSB of outer race faults at large clearance: (a) bispectrum (b) coherence

The tapered bearing operated at zero clearance means that the inner race, outer race and rolling elements keep good contacting during the rotation. Therefore, the slippage in such case is minimal in three cases and the deterministic behaviour dominates the dynamic responses of the whole bearing. The successive 
impacts of the roller passing the defect results in the vibration signals with a high SNR. Consequently, the fault features are easy to extract from the vibration measurements. As shown in Figure 18, both demodulation approaches produce distinctive fundamental and high order harmonics at the characteristic frequencies, allowing the fault to be diagnosed without doubt. However, the amplitude of the fault signatures is at different levels. The amplitude of the theoretical fault frequency in the PLMSB is more than 200 times larger than that in the MSB. The fault information obtained by the PLMSB is much more obvious with a tiny noise floor. In this condition, the MSB still works very well because the slippage between bearing components is small and the periodic signals are the principle components in the vibration measurements.

(a) PL-MSB Slice
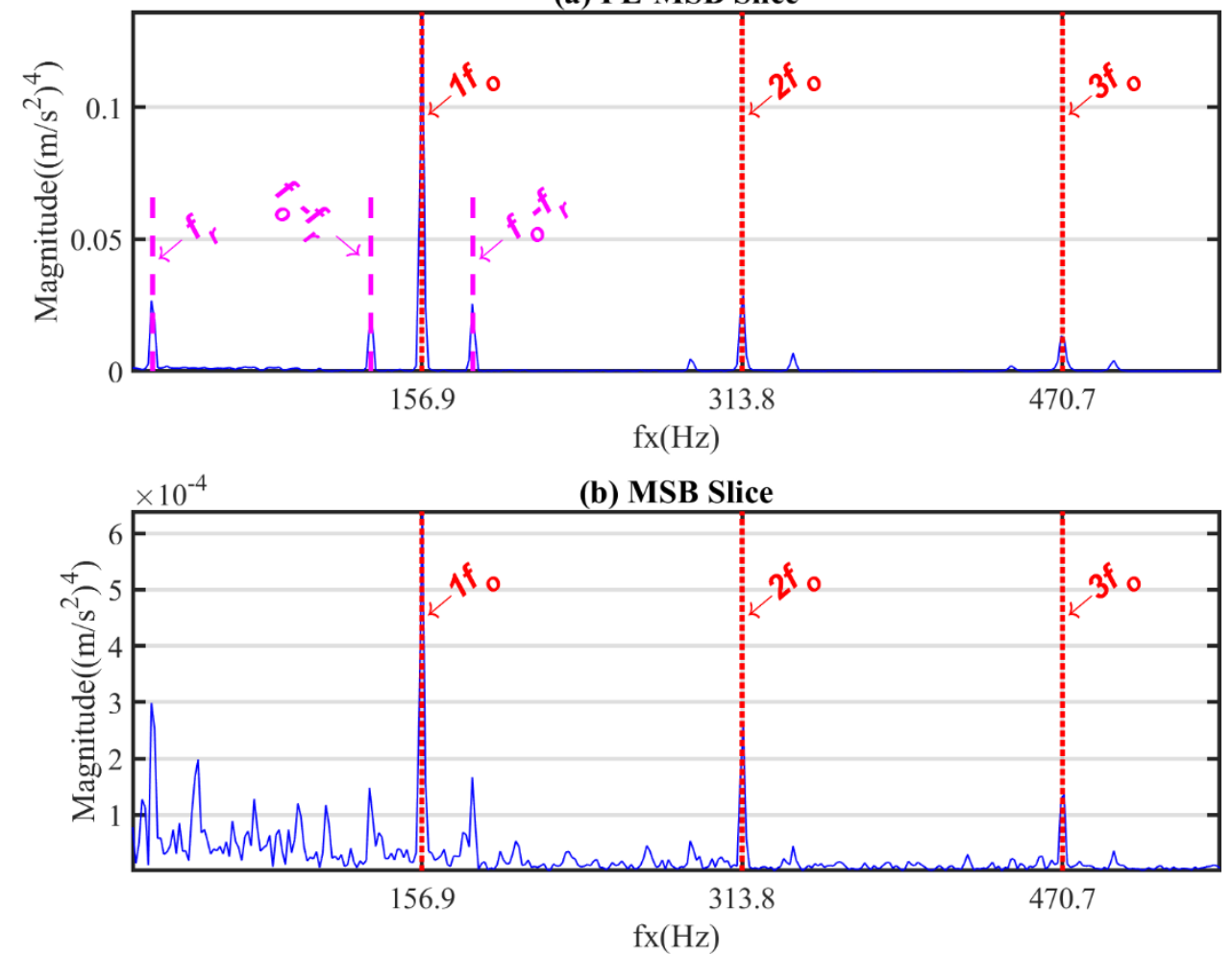

Figure 18 Slice of bispectrum for the outer race fault at zero clearance: (a)PL-MSB slice (b) MSB slice

Figure 19 (a) and (b) present the results of PL-MSB slice and MSB slice respectively for the vibration signals when the bearing is operated under medium clearance. The clearance between the bearing elements lead to the random slippage as well as the nonuniform impacts and hence, the fault signatures are difficult to be extracted. The results from the PL-MSB slice and MSB slice show that the fault features, both fundamental fault frequency and high order harmonics, have a much lower amplitude than that in the case of zero clearance. The MSB slice still has a sparse spectrum, which demonstrates that the random slippage in this working condition is not very high and conventional Fourier transform based MSB is powerful in this condition. The PL-MSB gives a better diagnosis result which illustrates that the proposed approach has good capability to process the cyclostationary bearing signals. 
(a) PL-MSB Slice
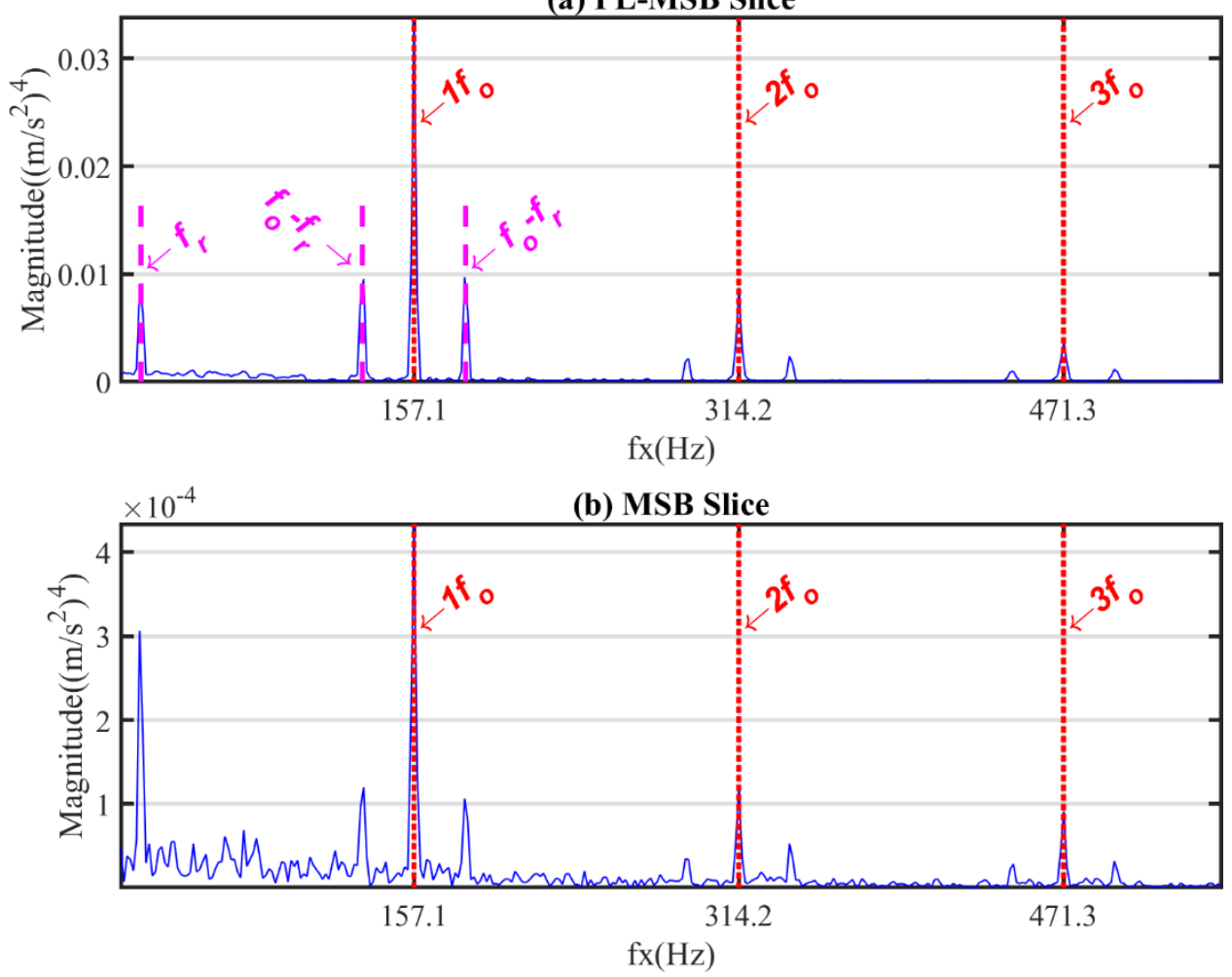

Figure 19 Slice of bispectrum for the outer race fault at medium clearance: (a)PL-MSB slice (b) MSB slice

The most challenging signals are obtained from the case of faulty bearings at large clearance. The components of the tapered bearings have no preload to keep them running at contacting conditions. Consequently, the random slippage of the tapered bearing during the rotation is considered to be greatest in the three cases. Additionally, the collisions between the seeded scratch and rollers are unstable due to the absence of the preload. The increase of the internal clearance also leads to the decrease of the contact length between the defect and the rollers, which further degenerates the SNR of the vibration signals. Figure 20 illustrates the concise results of the PL-MSB and MSB. The PL-MSB slice obviously discloses the first three harmonics of the theoretical outer ring fault frequency while the MSB only shows the first harmonic. The conventional MSB results are not convincible enough because the random peaks in the spectrum. The bicoherence of MSB in the Figure 17 (b) also denotes that the fault information in the MSB slice is not reliable because the coherence is very low. The PL-MSB supplies the accurate and reliable diagnostic results and consequently the performance of the PL-MSB is further verified to be effective for the bearing cyclostationary signals. 

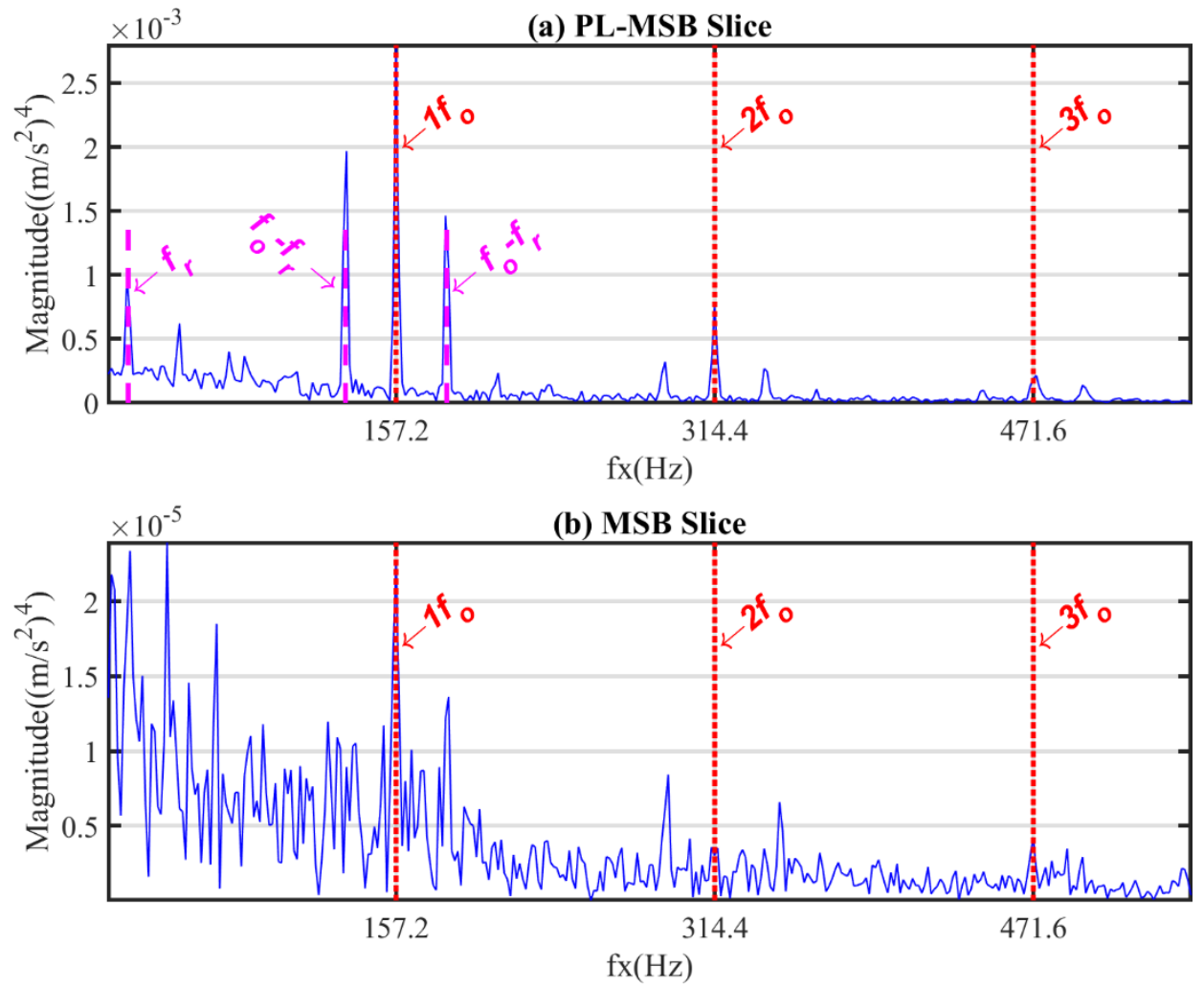

Figure 20 Slice of bispectrum for the outer race fault at large clearance: (a)PL-MSB slice (b) MSB slice

\section{Discussion and Conclusions}

The performance of MSB and PL-MSB is thoroughly investigated in the simulation studies. The MSB is not good at processing cyclostationary signals and the PL-MSB is capable in various slippage cases. In experimental studies, due to the randomness and uncertainty, the slippage between components is difficult to control and quantify. The conventional MSBs in experimental studies become worse along with the increase of the bearing clearance. These results tell that the deterministic components relating to the faults in the vibration signals decrease with the increase of the bearing clearance. The performance of the conventional MSB decays with the increase of the cyclostationary degree. The successful diagnosis of the bearing faults using conventional MSB depends on the strength of deterministic components relating to the faults. In contrast, the PL-MSB shows satisfied results in all experimental cases, which demonstrates the effectiveness of the proposed method upon the cyclostationary bearing fault signals.

The early fault detection and diagnosis is import, which can increase the reliability, productivity and safety of the machines. However, due to the random slippage, the theoretically periodic signals are distorted to be nonstationary, which makes the conventional methods no longer effective. To tackle the phase errors, a compensation method is proposed to eliminate the phase errors in the modulation signals. The phase linearisation can sufficiently suppress the phase noise and thus leads to the reconstruction signal approaching to a periodic one. The phase linearisation can effectively extend the application range of the Modulation Signal Bispectrum into the nonstationary signal processing. The PL-MSB can suppress the phase noise in the bearing vibration signals due to the random slippage and eliminate the background noise in the amplitude. Consequently the PL-MSB allows accurate fault diagnosis at the extremely bad condition with an SNR of $-22 \mathrm{~dB}$ and random slippage of $2 \%$ in the simulation study. Based on the experimental investigation, the proposed approach can detect and diagnose the bearing fault at various working conditions with sufficient confidence. The proposed approach is proved to be accurate and robust in detecting and diagnosing the incipient bearing faults. 


\section{Acknowledgment}

The authors would like to acknowledge the support from the China Scholarship Council (Grant No. 201608060042), the Natural Science Foundation of Guangdong Province (Grant No.2017A030313291) and Innovating major training projects of Beijing Institute of Technology, Zhuhai (Grant No. XKCQ2019-01). The authors would also appreciate the support from Dr Khalid Rabeyee in CEPE at University of Huddersfield.

\section{References}

1. Abdusslam SA. Detection and Diagnosis of Rolling Element Bearing Faults Using Time Encoded Signal Processing and Recognition. Doctoral, University of Huddersfield, http://eprints.hud.ac.uk/id/eprint/17806/ (2012, accessed 27 August 2017).

2. Chang Z, Jia Q, Yuan X, et al. Main failure mode of oil-air lubricated rolling bearing installed in high speed machining. Tribology International 2017; 112: 68-74.

3. Haneef MD, Randall RB, Smith WA, et al. Vibration and wear prediction analysis of IC engine bearings by numerical simulation. Wear 2017; 384-385: 15-27.

4. Nembhard AD, Sinha JK, Pinkerton AJ, et al. Combined vibration and thermal analysis for the condition monitoring of rotating machinery. Structural Health Monitoring 2014; 13: 281-295.

5. Kilundu B, Chiementin X, Duez J, et al. Cyclostationarity of Acoustic Emissions (AE) for monitoring bearing defects. Mechanical Systems and Signal Processing 2011; 25: 2061-2072.

6. Fei C-W, Choy Y-S, Bai G-C, et al. Multi-feature entropy distance approach with vibration and acoustic emission signals for process feature recognition of rolling element bearing faults. Structural Health Monitoring 2018; 17: 156-168.

7. Daraz A, Alabied S, Smith A, et al. Detection and diagnosis of centrifugal pump bearing faults based on the envelope analysis of airborne sound signals. In: 2018 24th International Conference on Automation and Computing (ICAC). 2018. Epub ahead of print 6 September 2018. DOI: 10.23919/IConAC.2018.8749053.

8. Zhen D, Guo J, Xu Y, et al. A Novel Fault Detection Method for Rolling Bearings Based on NonStationary Vibration Signature Analysis. Sensors 2019; 19: 3994.

9. Darlow MS, Badgley RH, Hogg GW. Application of High-Frequency Resonance Techniques for Bearing Diagnostics in Helicopter Gearboxes. No. MTI-74TR25. MECHANICAL TECHNOLOGY INC LATHAM NY, October 1974.

10. Antoni J, Randall RB. The spectral kurtosis: application to the vibratory surveillance and diagnostics of rotating machines. Mechanical Systems and Signal Processing 2006; 20: 308-331.

11. Gu F, Tian X, Chen Z, et al. Fault severity diagnosis of rolling element bearings based on kurtogram and envelope analysis. Institute of Research Engineers and Doctors, 2014.

12. Lei Y, Lin J, He Z, et al. Application of an improved kurtogram method for fault diagnosis of rolling element bearings. Mechanical Systems and Signal Processing 2011; 25: 1738-1749.

13. Smith WA, Fan Z, Peng Z, et al. Optimised Spectral Kurtosis for bearing diagnostics under electromagnetic interference. Mechanical Systems and Signal Processing 2016; 75: 371-394.

14. Mahvash A, Lakis AA. Application of cyclic spectral analysis in diagnosis of bearing faults in complex machinery. Tribology Transactions 2015; 58: 1151-1158.

15. Antoni J. Cyclic spectral analysis in practice. Mechanical Systems and Signal Processing 2007; 21: 597-630.

16. Randall RB, Antoni J, Chobsaard S. The relationship between spectral correlation and envelope analysis in the diagnostics of bearing faults and other cyclostationary machine signals. Mechanical Systems and Signal Processing 2001; 15: 945-962.

17. Ha JM, Youn BD, Oh H, et al. Autocorrelation-based time synchronous averaging for condition monitoring of planetary gearboxes in wind turbines. Mechanical Systems and Signal Processing 2016; 70-71: 161-175.

18. Bechhoefer E, Kingsley M. A Review of Time Synchronous Average Algorithms. In: Annual conference of the prognostics and health management society. 2009, pp. 1-10. 
19. Combet F, Gelman L. An automated methodology for performing time synchronous averaging of a gearbox signal without speed sensor. Mechanical Systems and Signal Processing 2007; 21: 2590-2606.

20. $\mathrm{Hu} \mathrm{Y,} \mathrm{Tu} \mathrm{X,} \mathrm{Li} \mathrm{F,} \mathrm{et} \mathrm{al.} \mathrm{An} \mathrm{adaptive} \mathrm{and} \mathrm{tacholess} \mathrm{order} \mathrm{analysis} \mathrm{method} \mathrm{based} \mathrm{on} \mathrm{enhanced}$ empirical wavelet transform for fault detection of bearings with varying speeds. Journal of Sound and Vibration 2017; 409: 241-255.

21. Wang Y, Xu G, Luo A, et al. An online tacholess order tracking technique based on generalized demodulation for rolling bearing fault detection. Journal of Sound and Vibration 2016; 367: 233249.

22. Cao H, He D, Xi S, et al. Vibration signal correction of unbalanced rotor due to angular speed fluctuation. Mechanical Systems and Signal Processing 2018; 107: 202-220.

23. $\mathrm{Lu} \mathrm{S}, \mathrm{He} \mathrm{Q}$, Zhao J. Bearing fault diagnosis of a permanent magnet synchronous motor via a fast and online order analysis method in an embedded system. Mechanical Systems and Signal Processing 2018; 113: 36-49.

24. He G, Ding K, Li W, et al. A novel order tracking method for wind turbine planetary gearbox vibration analysis based on discrete spectrum correction technique. Renewable Energy 2016; 87: 364-375.

25. Lu S, He Q, Wang J. A review of stochastic resonance in rotating machine fault detection. Mechanical Systems and Signal Processing 2019; 116: 230-260.

26. He Q, Wu E, Pan Y. Multi-Scale Stochastic Resonance Spectrogram for fault diagnosis of rolling element bearings. Journal of Sound and Vibration 2018; 420: 174-184.

27. Hou S, Li Y, Wang Z. A resonance demodulation method based on harmonic wavelet transform for rolling bearing fault diagnosis. Structural Health Monitoring 2010; 9: 297-308.

28. Cui H, Qiao Y, Yin Y, et al. An investigation on early bearing fault diagnosis based on wavelet transform and sparse component analysis. Structural Health Monitoring 2017; 16: 39-49.

29. Xu Y, Zhen D, Gu JX, et al. Autocorrelated Envelopes for early fault detection of rolling bearings. Mechanical Systems and Signal Processing 2021; 146: 106990.

30. Gu F, Shao Y, Hu N, et al. Motor current signal analysis using a modified bispectrum for machine fault diagnosis. In: 2009 ICCAS-SICE. 2009, pp. 4890-4895.

31. Tian X, Xi Gu J, Rehab I, et al. A robust detector for rolling element bearing condition monitoring based on the modulation signal bispectrum and its performance evaluation against the Kurtogram. Mechanical Systems and Signal Processing 2018; 100: 167-187.

32. Rehab I, Tian X, Gu F, et al. The fault detection and severity diagnosis of rolling element bearings using modulation signal bispectrum. In: 11th International Conference on Condition Monitoring and Machinery Failure Prevention Technologies. Manchester, UK, 2014.

33. Zhang R, Gu F, Mansaf H, et al. Gear wear monitoring by modulation signal bispectrum based on motor current signal analysis. Mechanical Systems and Signal Processing 2017; 94: 202-213.

34. Gu F, Wang T, Alwodai A, et al. A new method of accurate broken rotor bar diagnosis based on modulation signal bispectrum analysis of motor current signals. Mechanical Systems and Signal Processing 2015; 50-51: 400-413.

35. Gu F, Shao Y, Hu N, et al. Electrical motor current signal analysis using a modified bispectrum for fault diagnosis of downstream mechanical equipment. Mechanical Systems and Signal Processing 2011; 25: 360-372.

36. Hamomd $\mathrm{O}$, Alabied $\mathrm{S}, \mathrm{Xu} \mathrm{Y}$, et al. Vibration based centrifugal pump fault diagnosis based on modulation signal bispectrum analysis. In: 2017 23rd International Conference on Automation and Computing (ICAC). 2017, pp. 1-5.

37. Randall RB, Antoni J, Chobsaard S. A comparison of cyclostationary and envelope analysis in the diagnostics of rolling element bearings. In: 2000 IEEE International Conference on Acoustics, Speech, and Signal Processing. Proceedings (Cat. No.00CH37100). 2000, pp. 3882-3885 vol.6.

38. Wang Y-F, Kootsookos PJ. Modeling of Low Shaft Speed Bearing Faults for Condition Monitoring. Mechanical Systems and Signal Processing 1998; 12: 415-426.

39. Patel VN, Tandon N, Pandey RK. A Dynamic Model for Vibration Studies of Deep Groove Ball Bearings Considering Single and Multiple Defects in Races. Journal of Tribology 2010; 132: 041101. 
40. Rehab I, Tian X, Gu F, et al. A study of diagnostic signatures of a deep groove ball bearing based on a nonlinear dynamic model. In: Automation and Computing (ICAC), 2015 21st International Conference on. IEEE, pp. 1-7.

41. Sawalhi N, Randall RB. Simulating Gear and Bearing Interactions in the Presence of Faults. Mechanical Systems and Signal Processing 2008; 22: 1924-1951.

42. Patil MS, Mathew J, Rajendrakumar PK, et al. A theoretical model to predict the effect of localized defect on vibrations associated with ball bearing. International Journal of Mechanical Sciences 2010; 52: 1193-1201. 


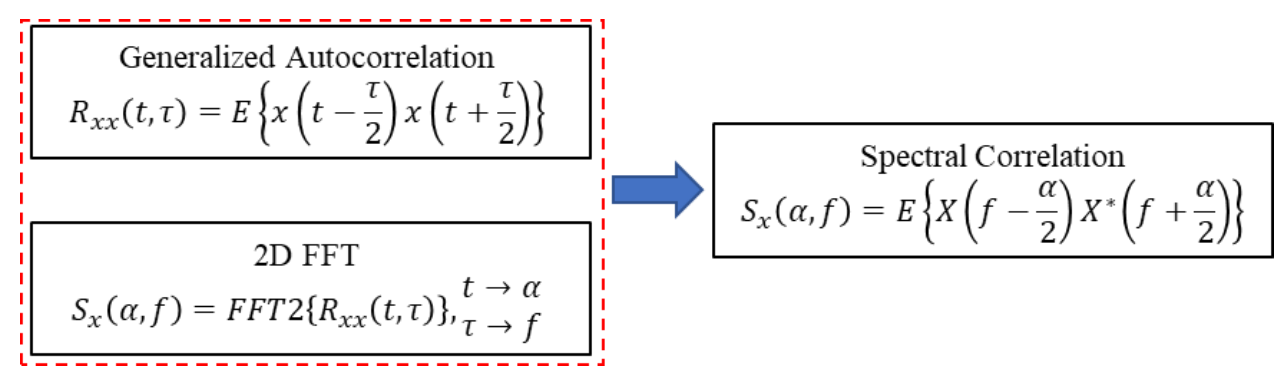

Figure 1 Theoretical background of Spectral Correlation

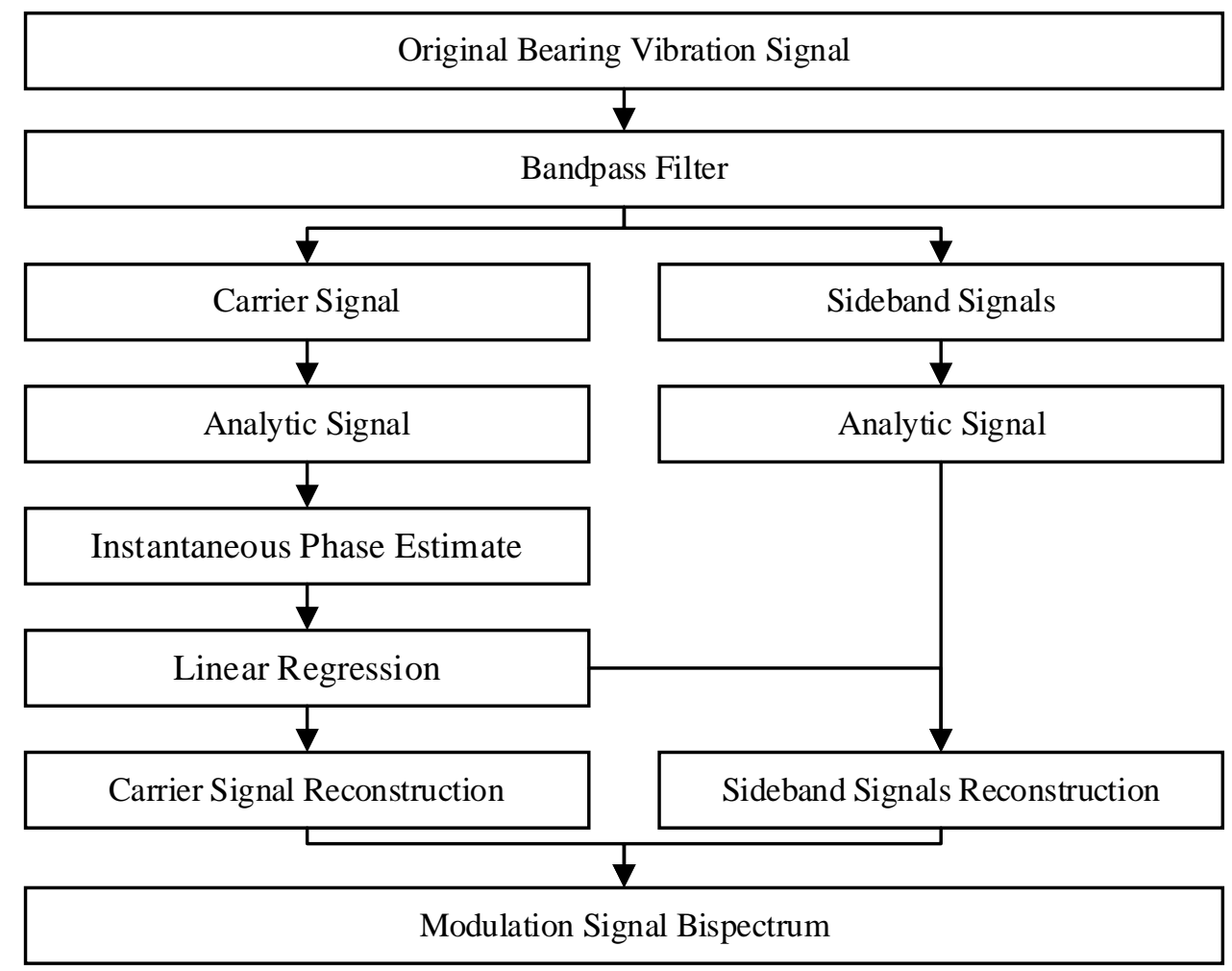

Figure 2 Flow chart of the PL-MSB method 
(a) Impact Series

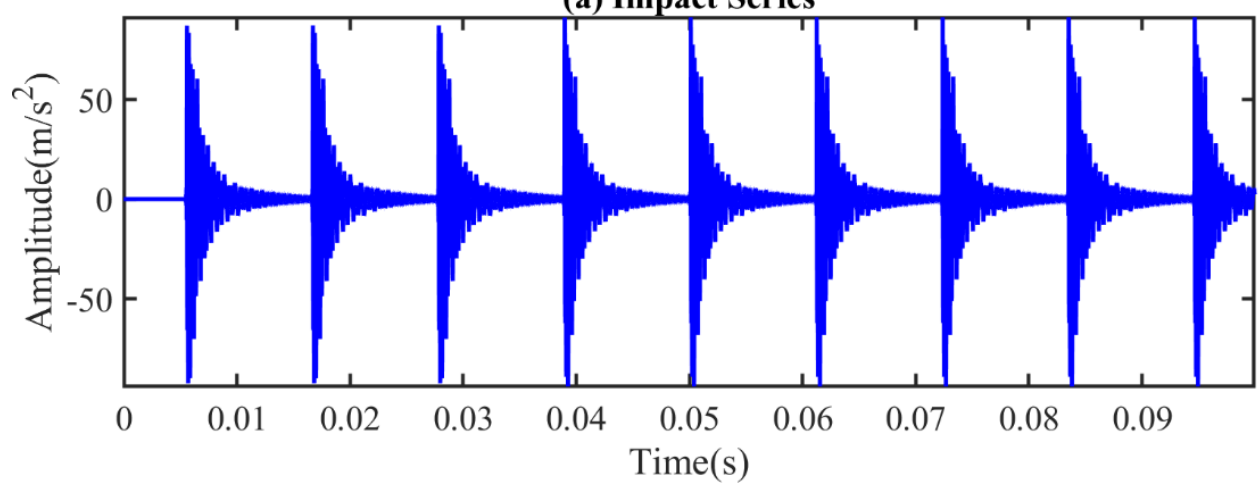

(b) Instantaneous Fault Frequency

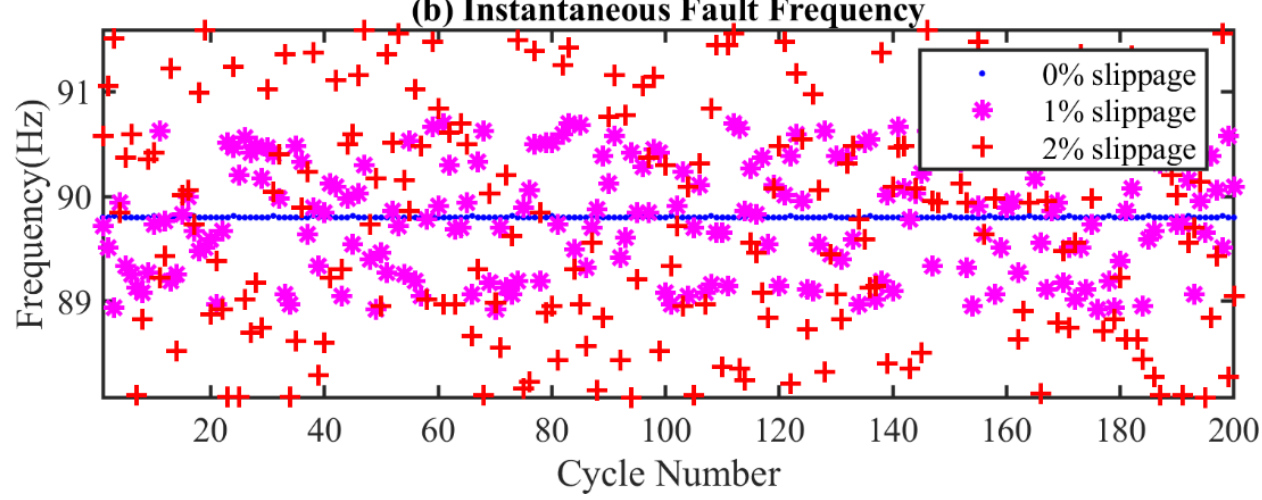

Figure 3 Simulated bearing outer race fault signals: (a) simulated bearing vibration with defects (b) fault periods varying due to slippage

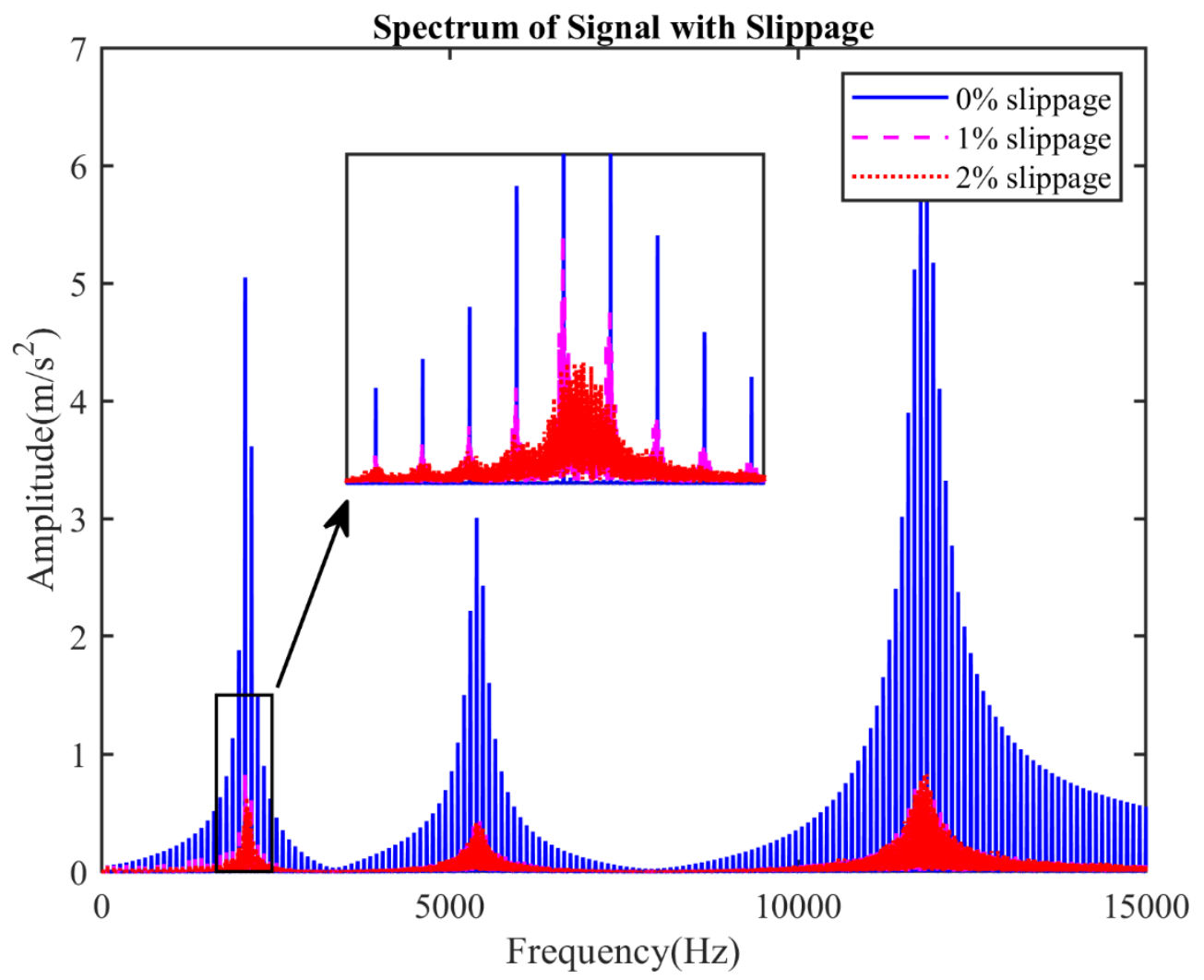

Figure 4 Amplitude spectra of simulated bearing outer race fault signals at different slippages 
(a) Time Waveform

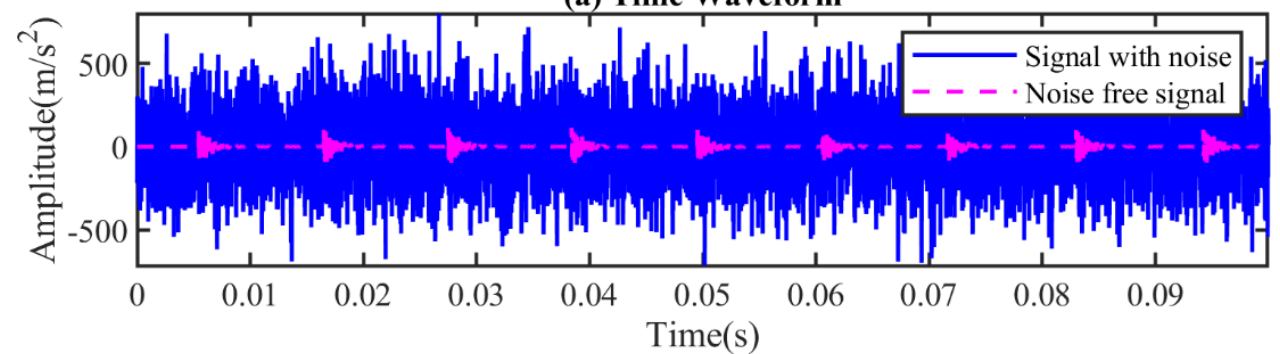

(b) Spectrum
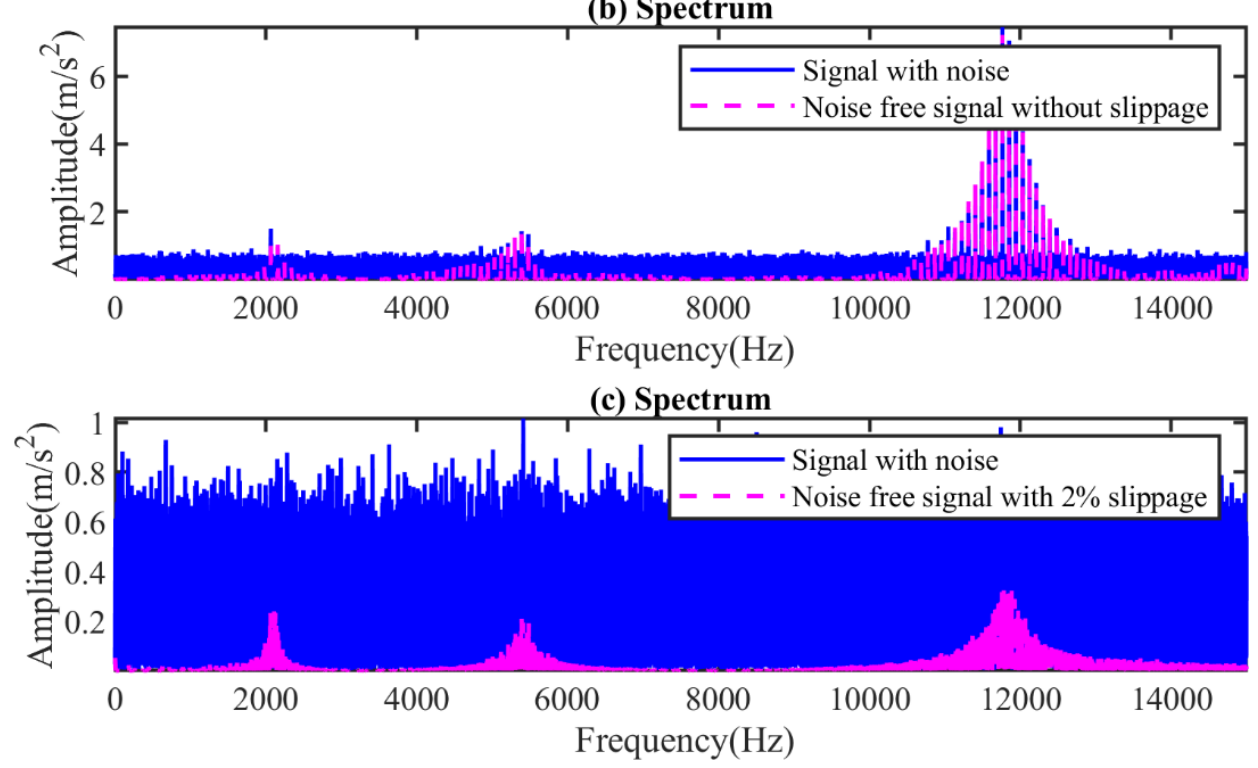

Figure 5 Simulated bearing outer race fault signals at the SNR of -22dB: (a) time waveforms with $2 \%$ slippage, (b) spectra of the signal without slippage, (c) spectra of the signal with $2 \%$ slippage

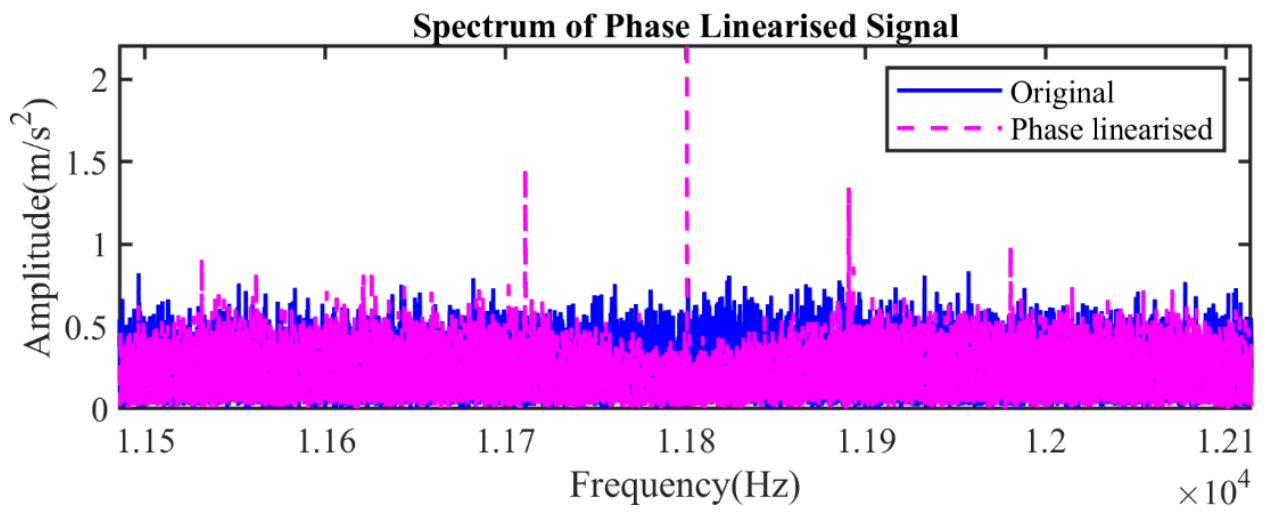

Figure 6 Phase linearised outer race fault signals at 2\% slippage and SNR-22dB 
(a) PL-MSB

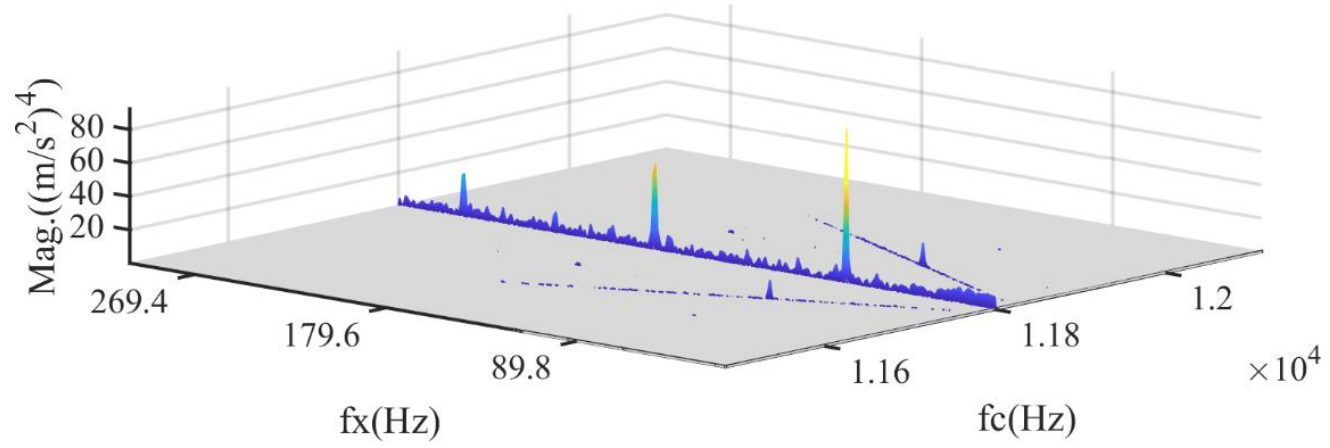

(b) PL-MSB Coherence

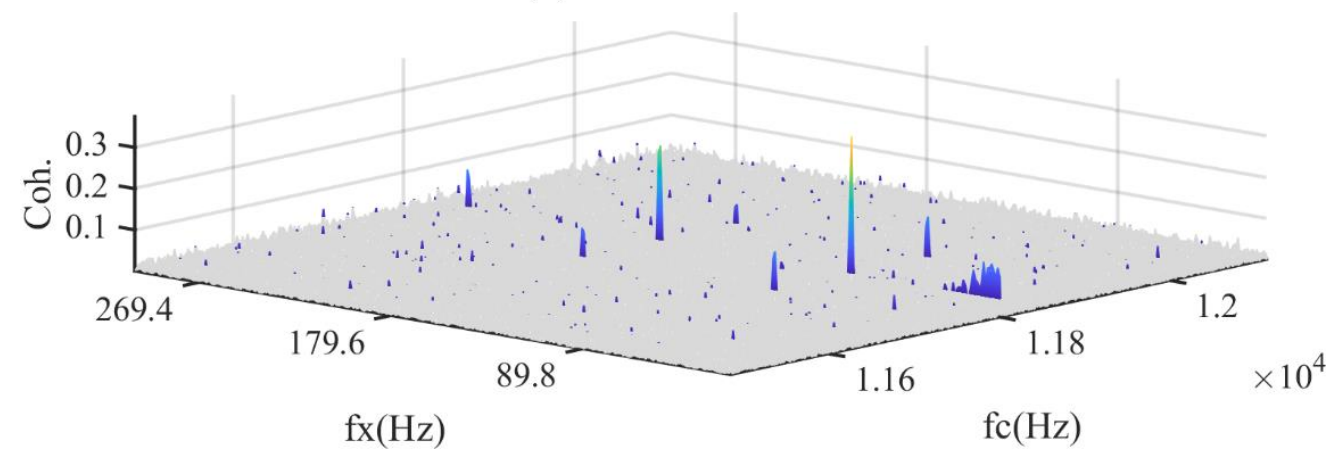

Figure 7 PL-MSB of outer race faults at 2\% slippage and SNR-22dB: (a) bispectrum (b) coherence (a) MSB

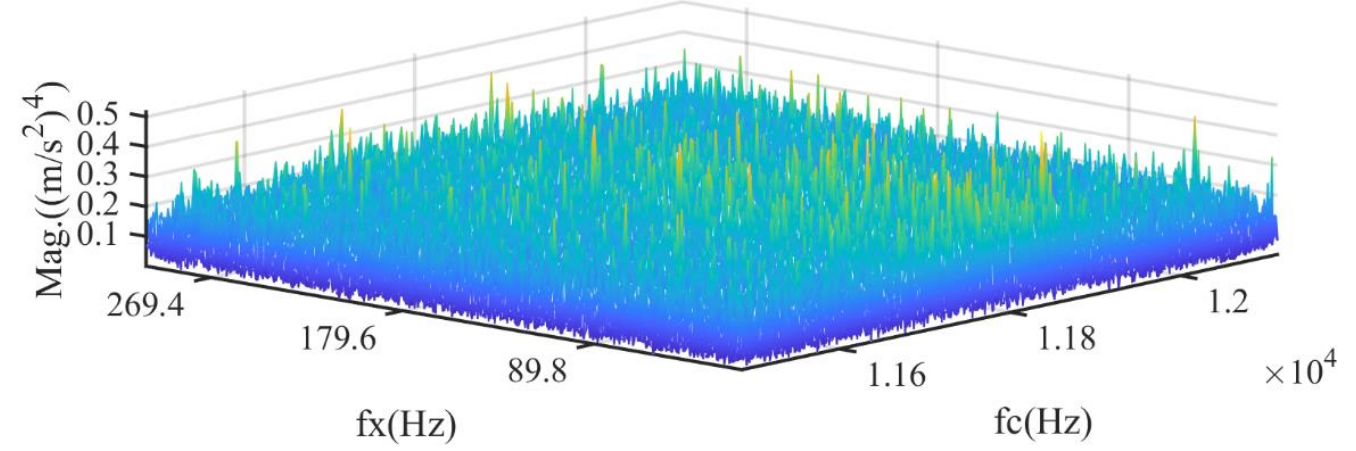

(b) MSB Coherence

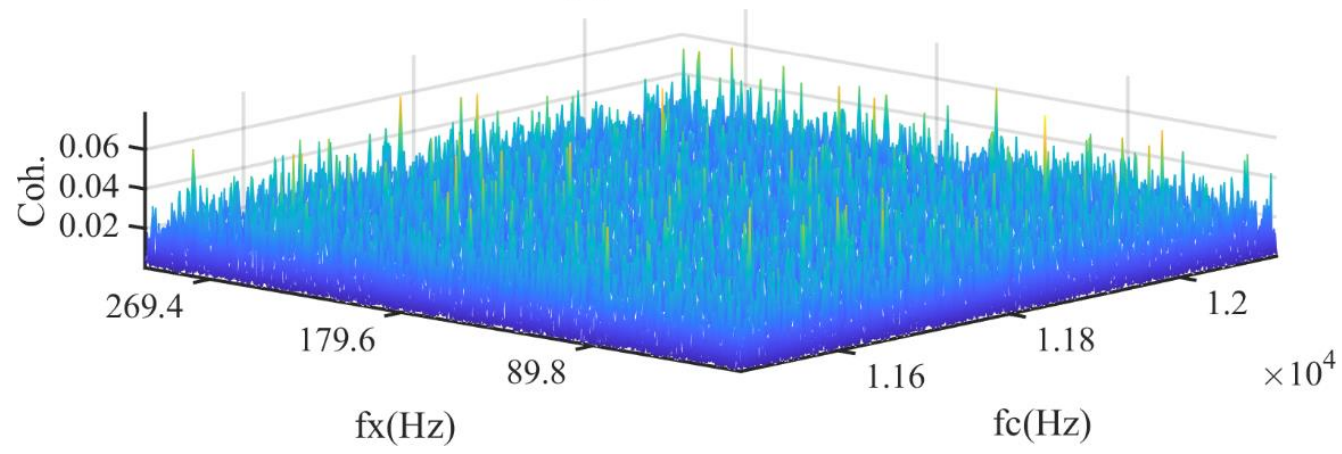

Figure 8 Conventional MSB of outer race faults at 2\% slippage and SNR-22dB: (a) bispectrum (b) coherence 
(a) PL-MSB Slice

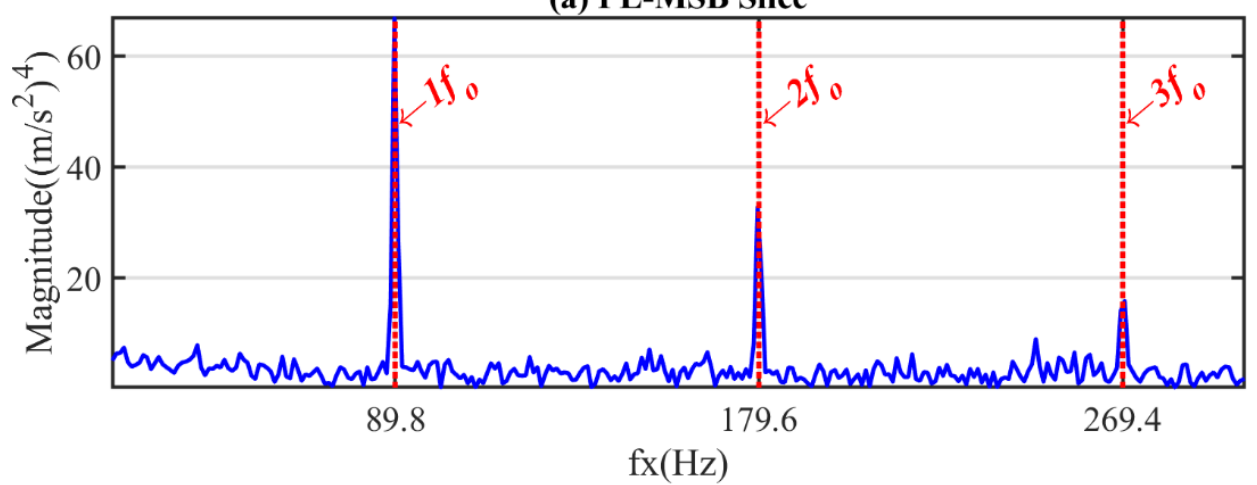

(b) MSB Slice

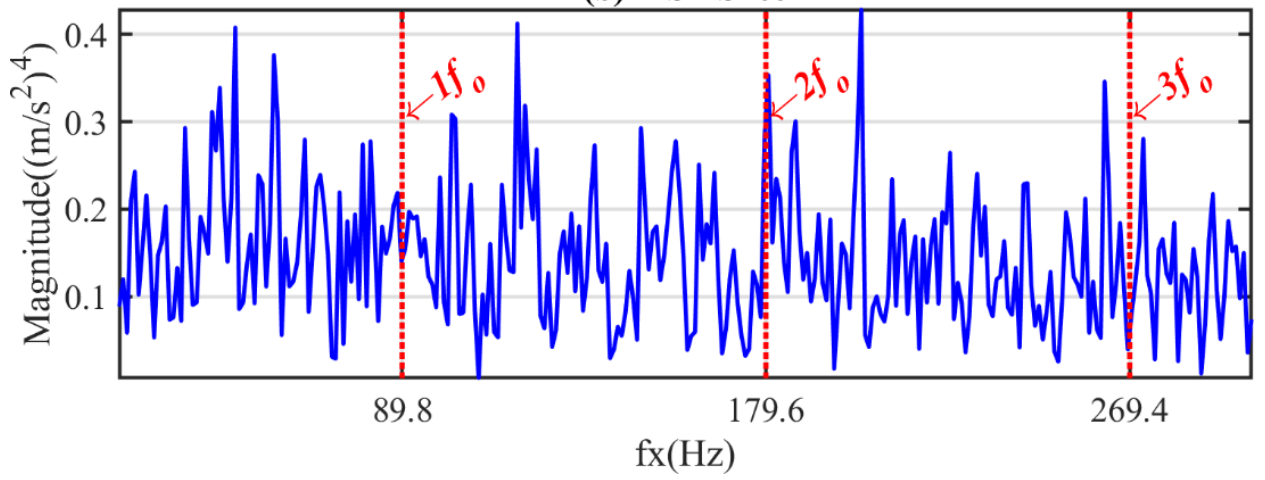

Figure 9 Slice of bispectrum for the outer race fault at 2\% slippage and SNR -22dB: (a)PL-MSB slice (b) MSB slice

(a) PL-MSB Slice

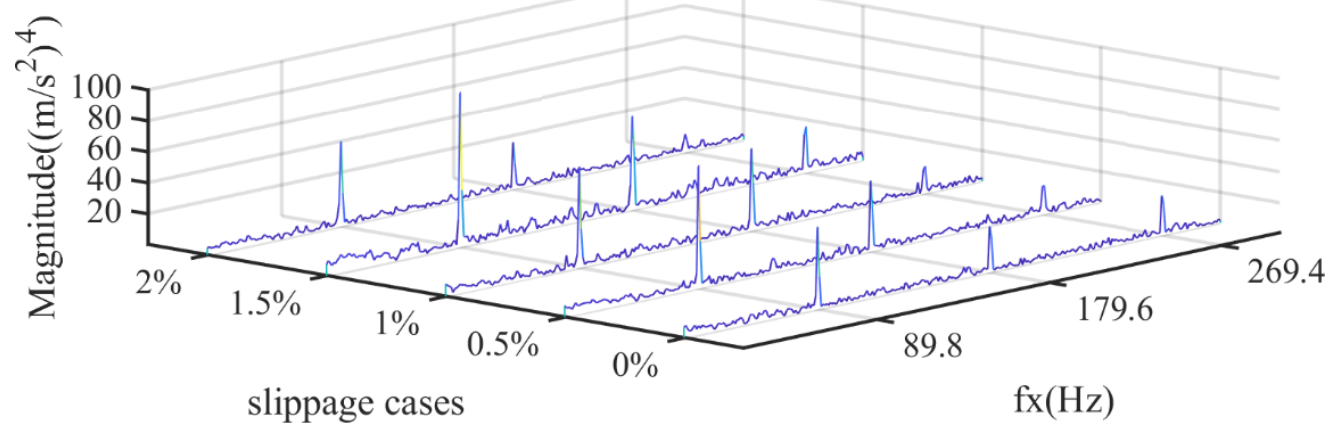

(b) MSB Slice

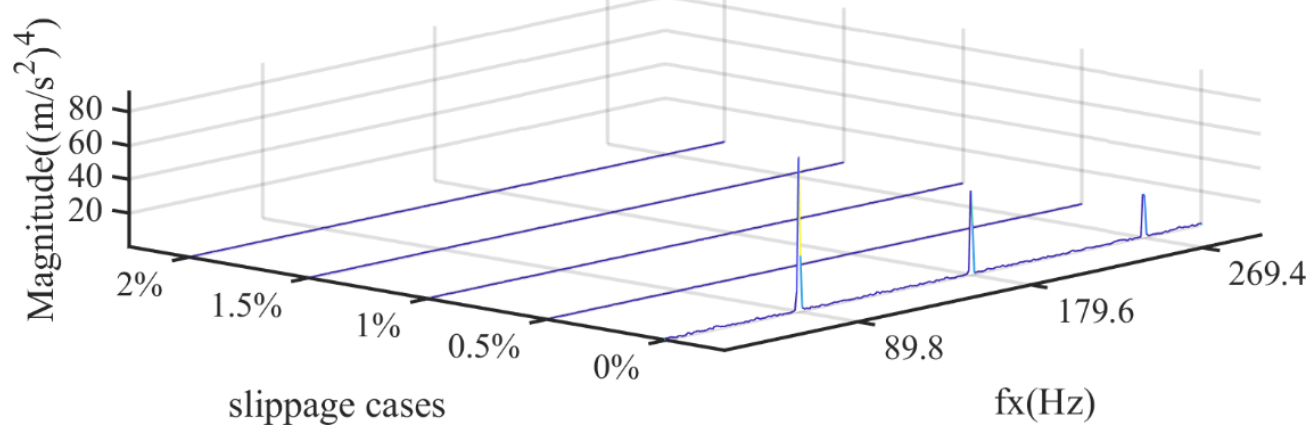

Figure 10 Diagnostic results of outer race faults at SNR-22dB: (a) PL-MSB slices (b) MSB slices 
(a) PL-MSB Slice

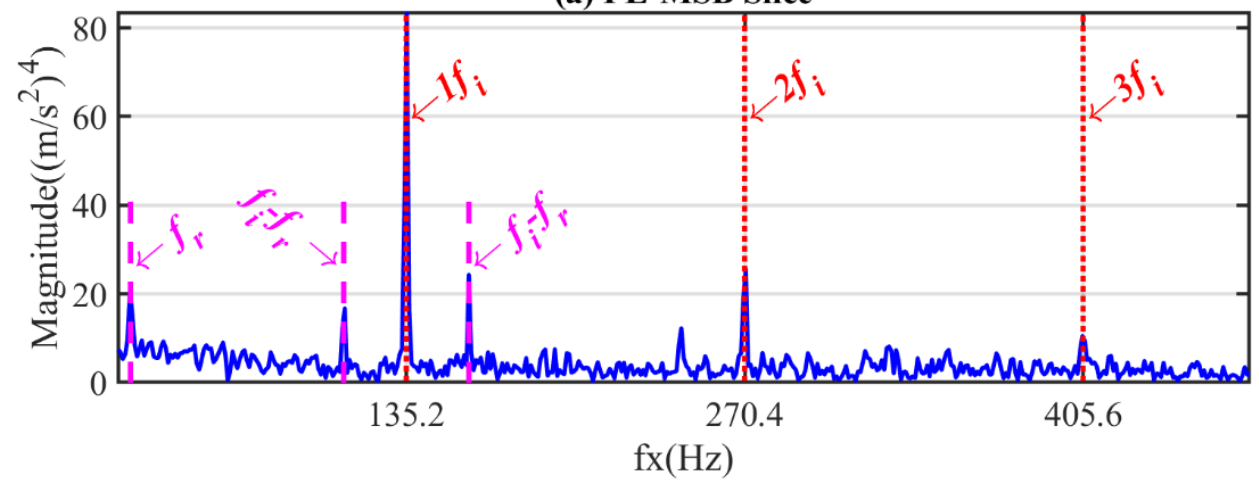

(b) MSB Slice

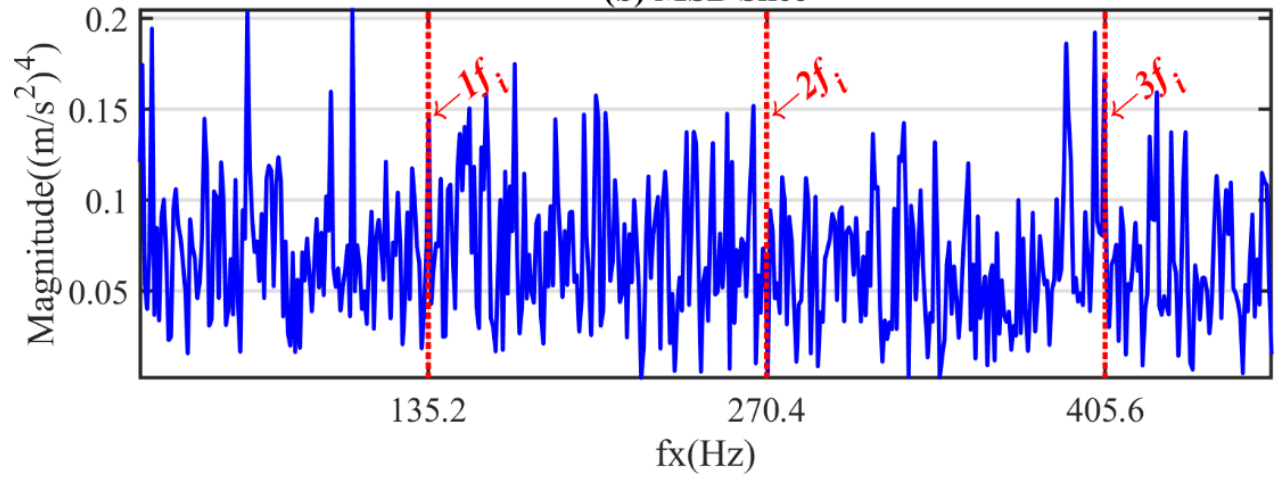

Figure 11 Slice of bispectrum for the inner race fault at 2\% slippage and SNR -22dB: (a)PL-MSB slice (b) MSB slice

(a) PL-MSB Slice

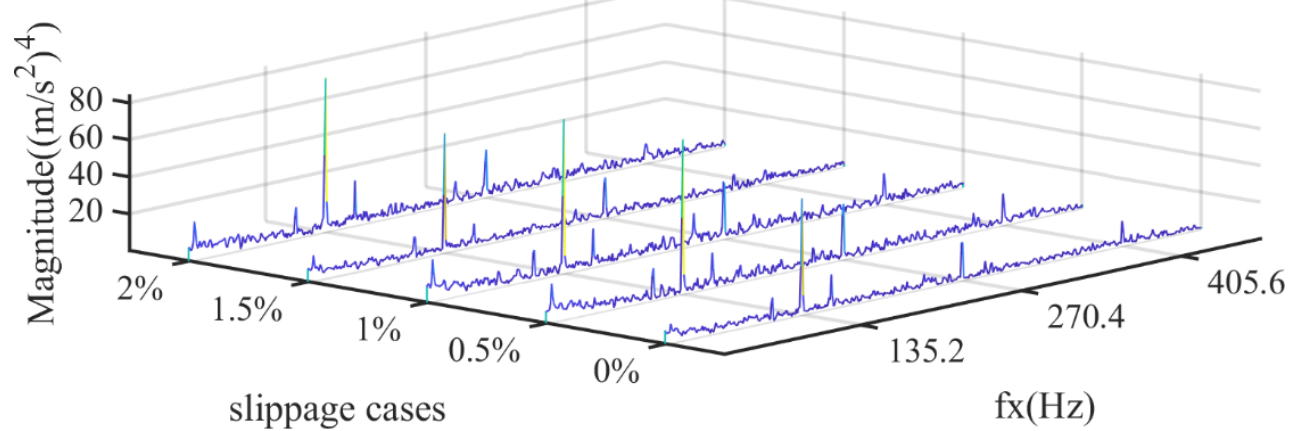

(b) MSB Slice

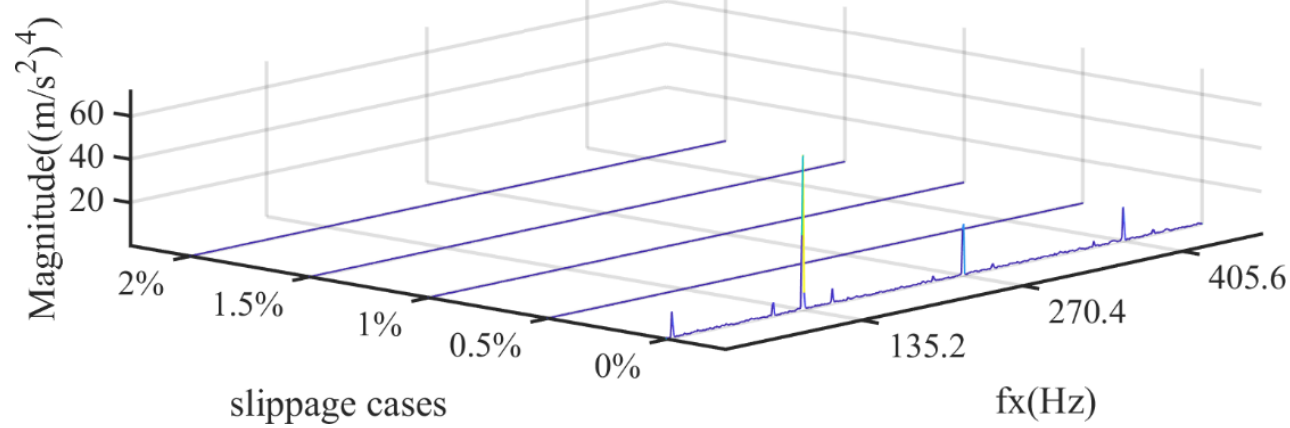

Figure 12 Diagnostic results of inner race faults at SNR-22dB: (a) PL-MSB slices (b) MSB slices 

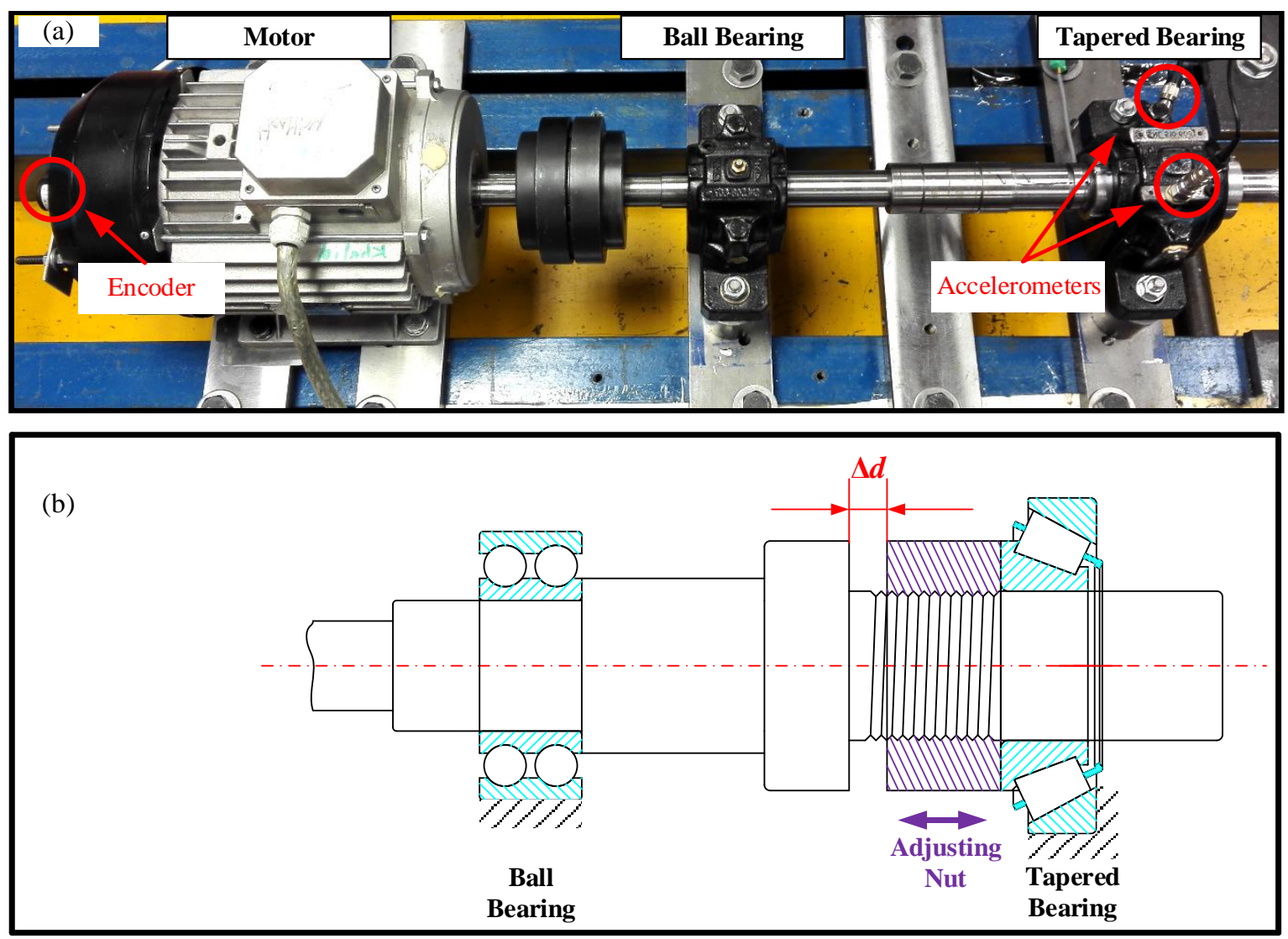

Figure 13 Test rig for the tapered roller bearing: (a) photograph, and (b) schematic diagram

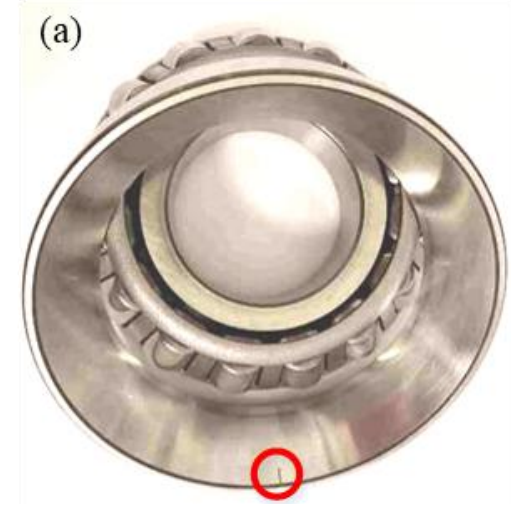

(b)

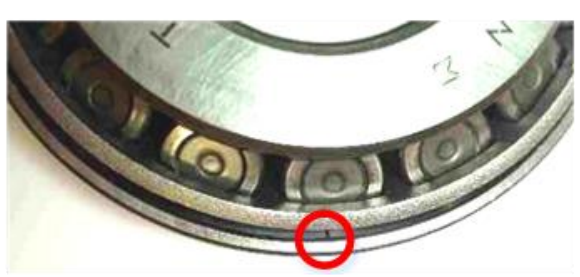

Figure 14 Photograph of the outer race fault of the bearing: (a) with bearing disassembled, and (b) with bearing assembled 
(a) Vibration of Tapered Bearing at Zero Clearance

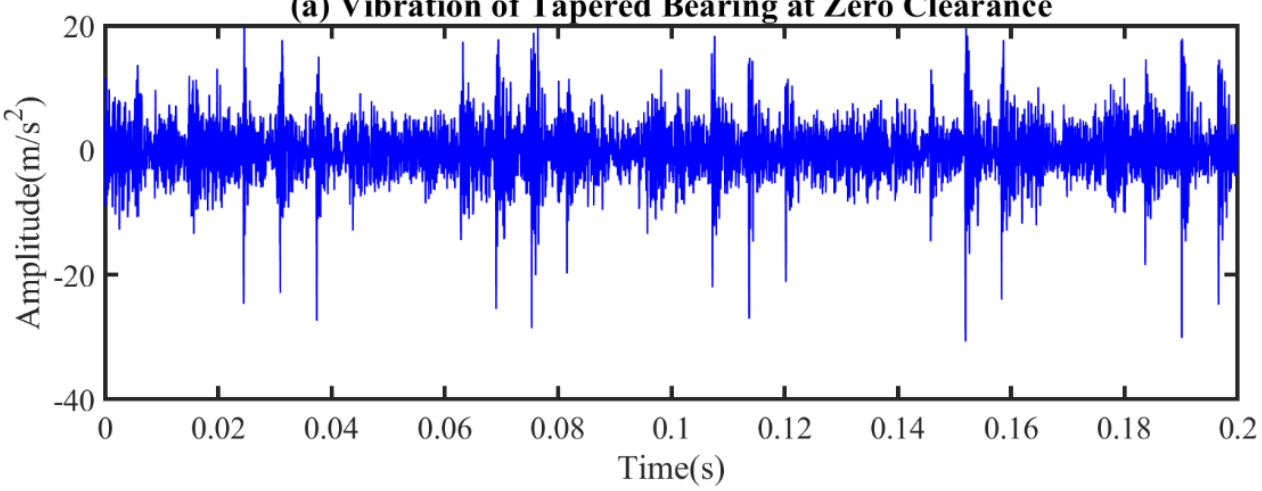

(b) Vibration Spectrum of Tapered Bearing at Zero Clearance

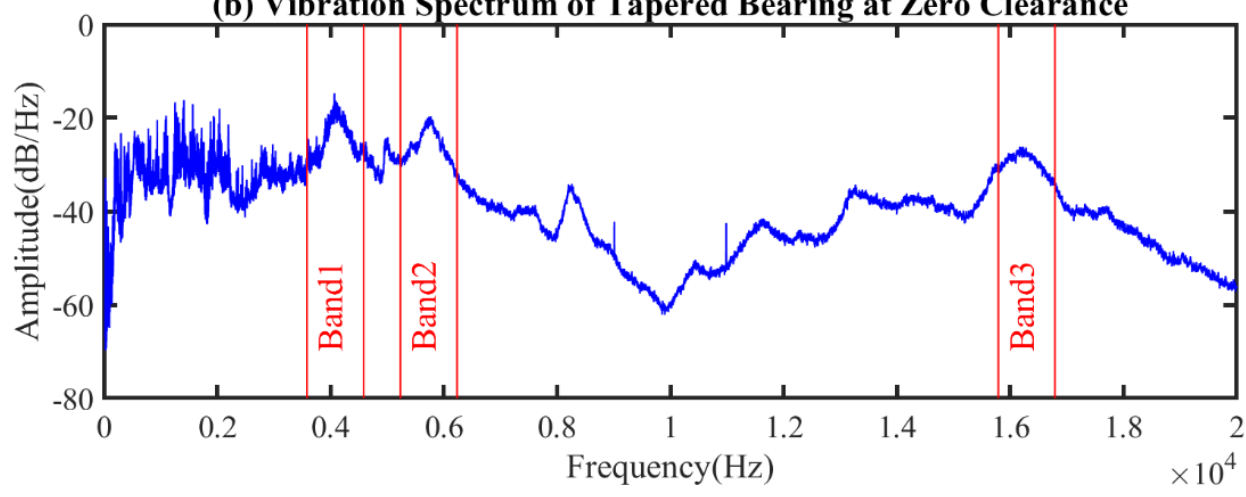

Figure 15 Vibration signals of the tapered bearing: (a) time domain signal of the tapered bearing at zero clearance, and (b) power spectrum density of (a)

(a) PL-MSB

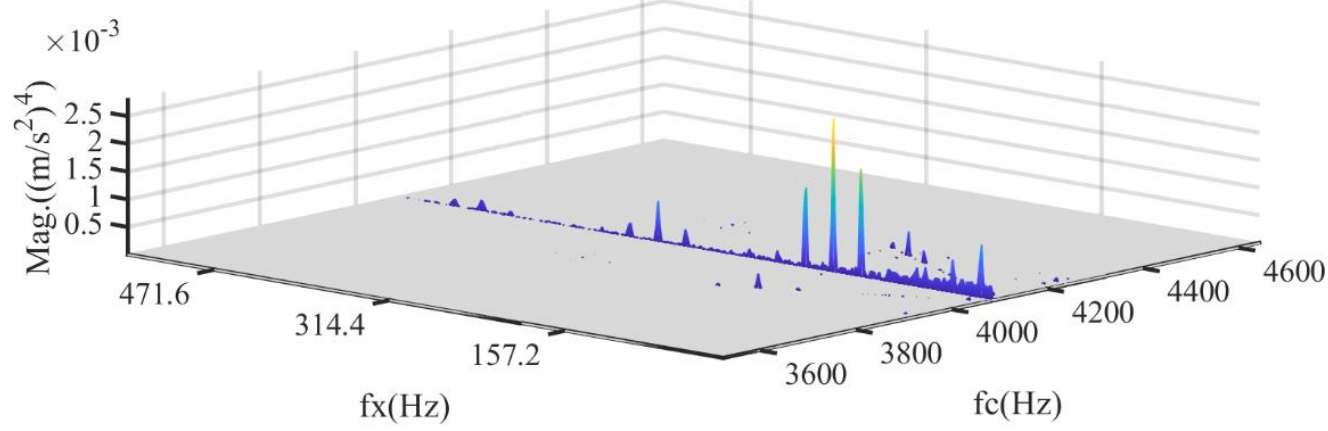

(b) PL-MSB Coherence

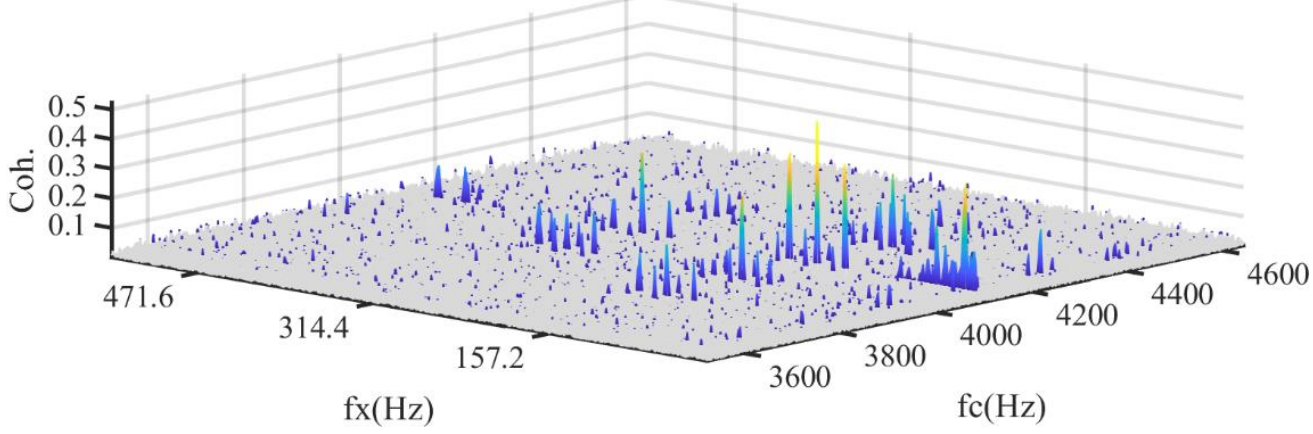

Figure 16 PL-MSB of outer race faults at large clearance: (a) bispectrum (b) coherence 
(a) MSB

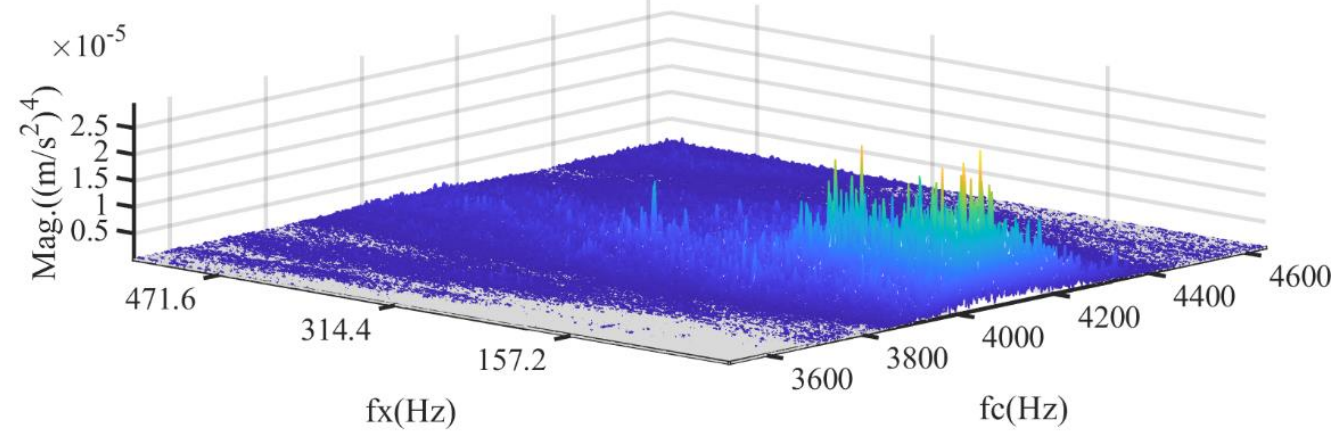

(b) MSB Coherence

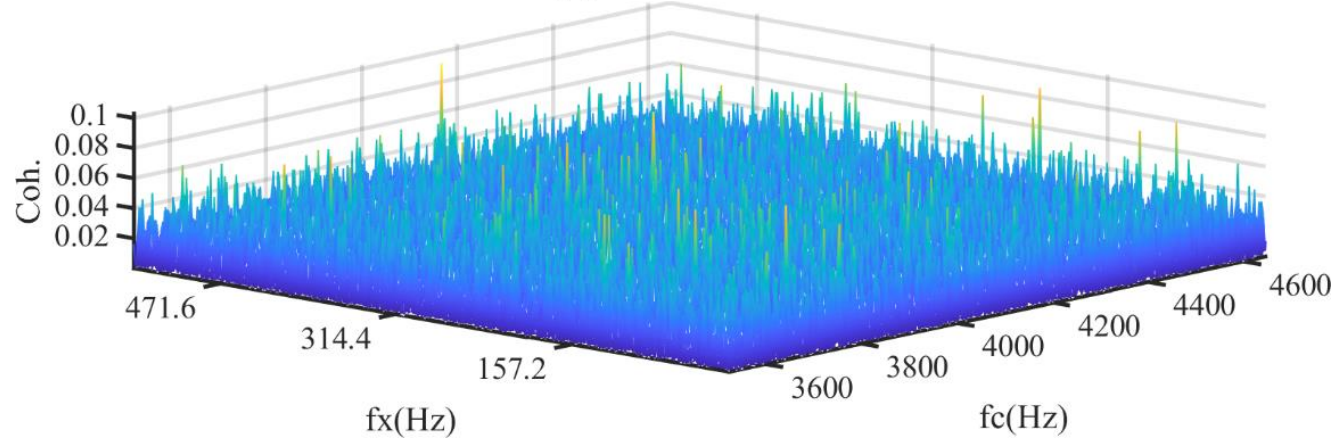

Figure 17 Conventional MSB of outer race faults at large clearance: (a) bispectrum (b) coherence
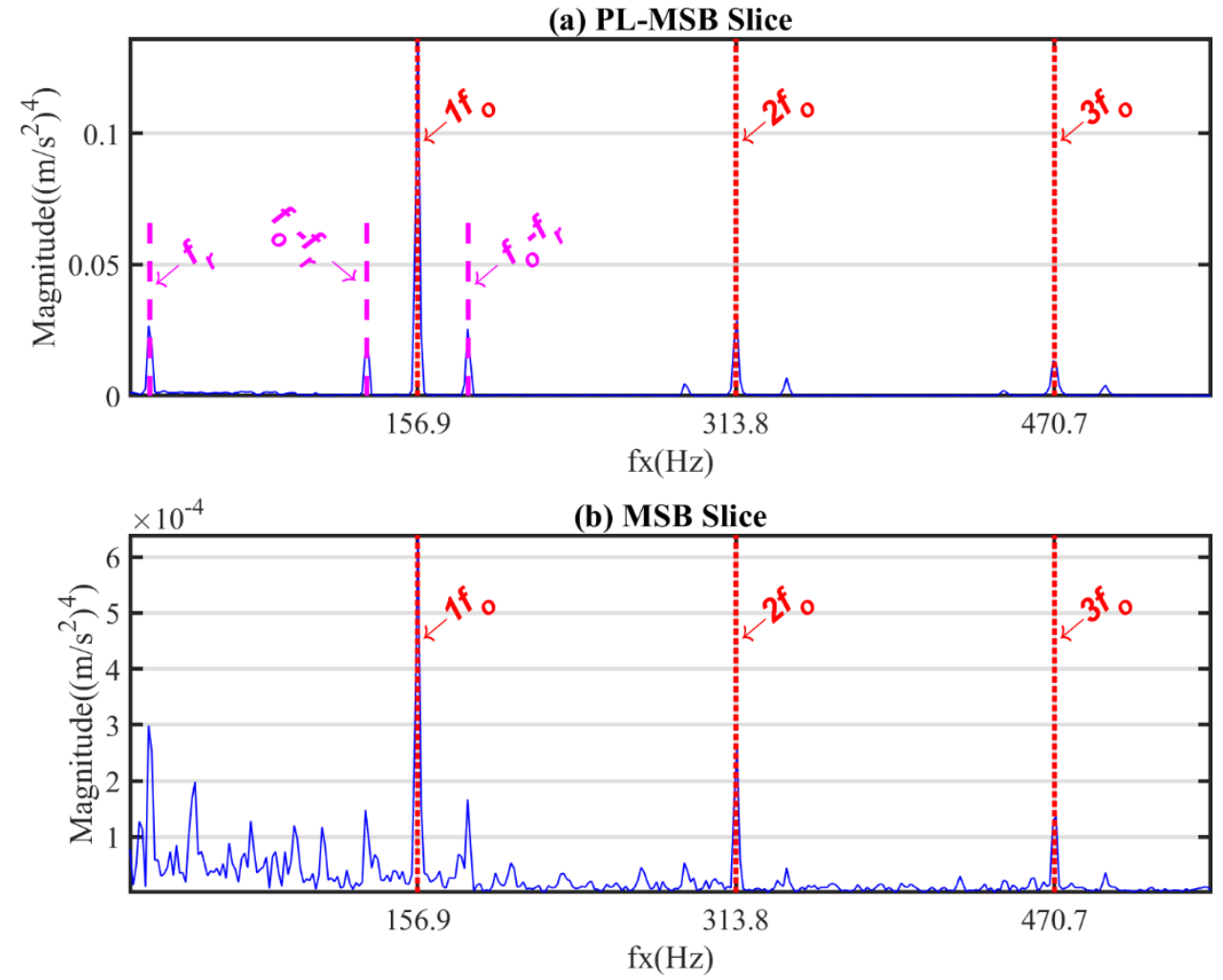

Figure 18 Slice of bispectrum for the outer race fault at zero clearance: (a)PL-MSB slice (b) MSB slice 
(a) PL-MSB Slice
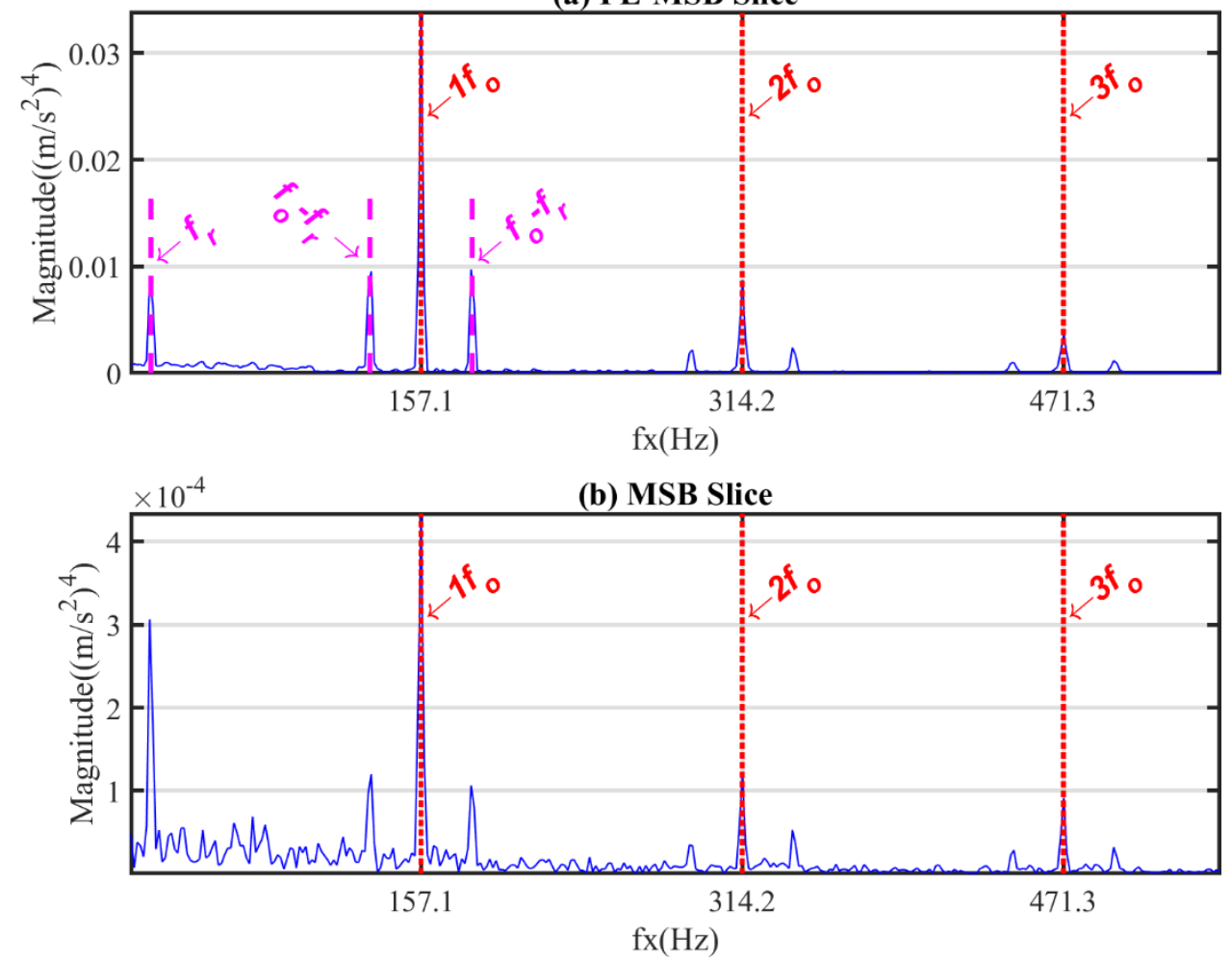

Figure 19 Slice of bispectrum for the outer race fault at medium clearance: (a)PL-MSB slice (b) MSB slice
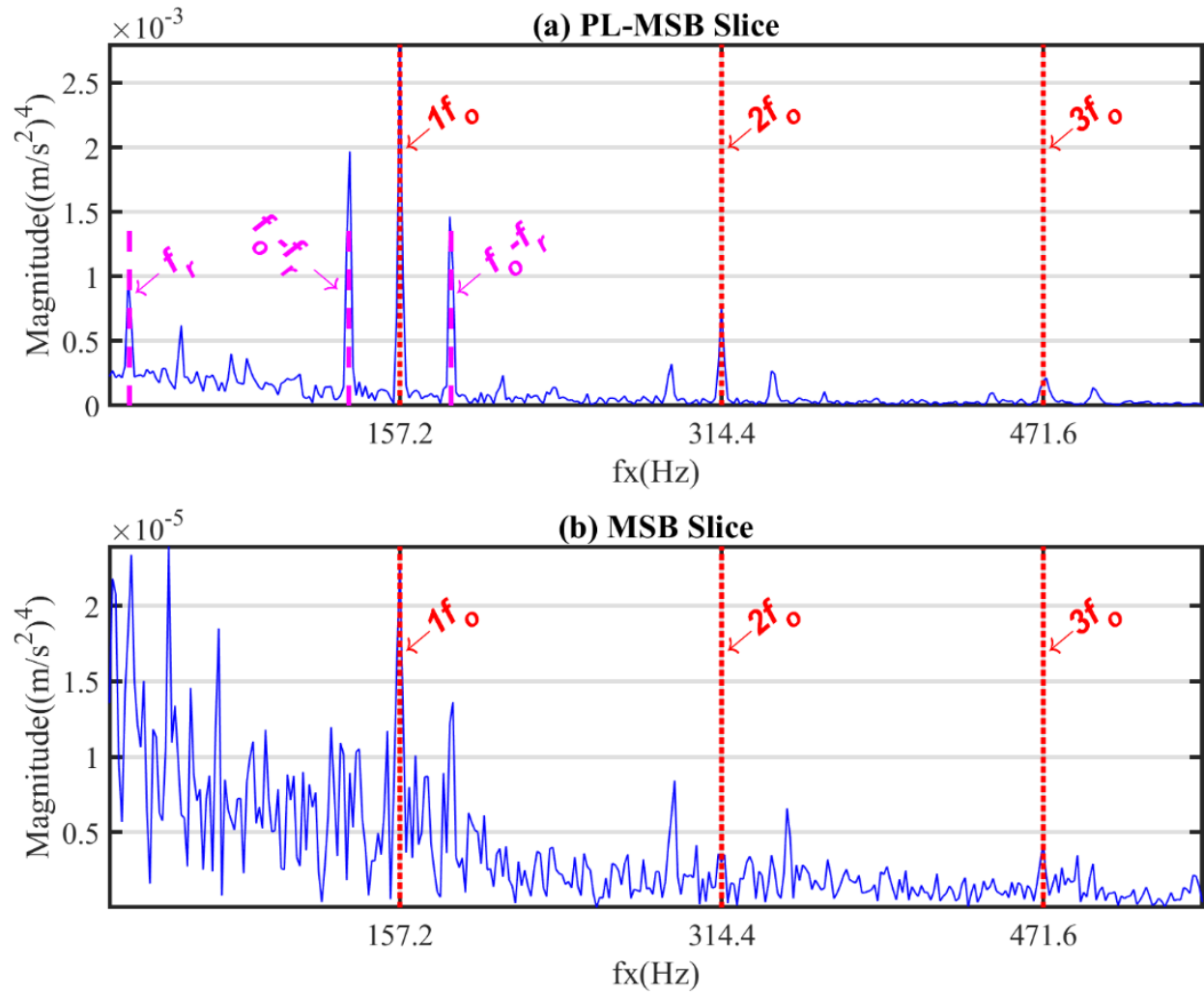

Figure 20 Slice of bispectrum for the outer race fault at large clearance: (a)PL-MSB slice (b) MSB slice 
Table 1 Key parameters of the simulated signals

\begin{tabular}{cc}
\hline Parameters & Value \\
Sampling Frequency & $96000 \mathrm{~Hz}$ \\
Sampling Time & $90 \mathrm{~s}$ \\
Outer Race Fault Frequency & $89.8 \mathrm{~Hz}$ \\
Inner Race Fault Frequency & $135.2 \mathrm{~Hz}$ \\
Natural Frequencies & $2100 \mathrm{~Hz}, 5400 \mathrm{~Hz}$ and $11800 \mathrm{~Hz}$ \\
\hline
\end{tabular}

Table 2 Bandpass filters

\begin{tabular}{cc}
\hline \multicolumn{2}{c}{ Table 2 Bandpass filters } \\
\hline Bandpass Filters & Frequency Bands \\
Carrier signal & {$[11753.6 \mathrm{~Hz}, 11846.4 \mathrm{~Hz}]$} \\
Lower sidebands 1 & {$[11660.8 \mathrm{~Hz}, 11753.6 \mathrm{~Hz}]$} \\
Lower sidebands 2 & {$[11568.0 \mathrm{~Hz}, 11660.8 \mathrm{~Hz}]$} \\
Lower sidebands 3 & {$[11475.2 \mathrm{~Hz}, 11568.0 \mathrm{~Hz}]$} \\
Upper sidebands 1 & {$[11846.4 \mathrm{~Hz}, 11939.2 \mathrm{~Hz}]$} \\
Upper sidebands 2 & {$[11939.2 \mathrm{~Hz}, 12032.0 \mathrm{~Hz}]$} \\
Upper sidebands 3 & {$[12032.0 \mathrm{~Hz}, 12124.8 \mathrm{~Hz}]$} \\
\hline
\end{tabular}

Table 3 Specifications of the data acquisition system

\begin{tabular}{lll}
\hline Item & Model & Key Specifications \\
\hline Date Acquisition Card & NI 6221 & $16-\mathrm{bit}, 250 \mathrm{kS} / \mathrm{s}$ overall \\
Charge Amplifier & B\&K 2635 & $0.2 \mathrm{~Hz}-100 \mathrm{kHz}$ with noise $<5 \times 10^{-3} \mathrm{pC}$ \\
Accelerometers & CA-YD-104T & $3.640 \mathrm{pC} / \mathrm{ms}^{-2}$ in $0.5 \mathrm{~Hz}-7000 \mathrm{~Hz}$ \\
Thermocouple & K type & $0-200^{\circ} \mathrm{C}$ range, $\pm 1.1^{\circ} \mathrm{C}$ \\
Encoder & RI32 & $100 \mathrm{ppr}$, max speed $6000 \mathrm{rpm}$ \\
Tapered Bearing & 31308 & $40 \mathrm{~mm}, 90 \mathrm{~mm}, 25.25 \mathrm{~mm}$ \\
\hline
\end{tabular}

Table 4 Test cases

\begin{tabular}{lll}
\hline Test Cases & Speed & $\boldsymbol{\Delta} \boldsymbol{d}$ \\
\hline Zero Clearance & $100 \%$ & $14.85 \mathrm{~mm}$ \\
Medium Clearance & $(1500 \mathrm{rpm})$ & $14.81 \mathrm{~mm}$ \\
Large Clearance & & $14.77 \mathrm{~mm}$ \\
\hline
\end{tabular}

